IMA Journal of Numerical Analysis (2016) Page 1 of 17

doi:10.1093/imanum/drnxxx

\title{
Pure vorticity formulation and Galerkin discretization for the Brinkman equations
}

\author{
VERÓNICA ANAYA† \\ GIMNAP, Departamento de Matemática, Universidad del Bío-Bío, Concepción, Chile \\ DAVID MORA $\ddagger$ \\ GIMNAP, Departamento de Matemática, Universidad del Bío-Bío, Concepción, Chile; and \\ Centro de Investigación en Ingeniería Matemática $\left(\mathrm{CI}^{2} \mathrm{MA}\right)$, Universidad de Concepción, \\ Concepción, Chile \\ AND \\ RICARDO RUIZ-BAIER $\S$ \\ Mathematical Institute, Oxford University, Andrew Wiles Building, Woodstock Road, OX2 \\ $6 G G$ Oxford, $U K$
}

[Received on September 20, 2016; revised on September 20, 2016]

\begin{abstract}
We introduce a new finite element method for the approximation of the three-dimensional Brinkman problem formulated in terms of the velocity, vorticity, and pressure fields. The proposed strategy exhibits the advantage that, at the continuous level, a complete decoupling of vorticity and pressure can be established under the assumption of sufficient regularity. The velocity is then obtained as a simple postprocess from vorticity and pressure, using the momentum equation. Well-posedness follows straightforwardly by the Lax-Milgram theorem. The Galerkin scheme is based on Nédélec and piecewise continuous finite elements of degree $k \geqslant 1$ for vorticity and pressure, respectively. The discrete setting uses the very same ideas as in the continuous case, and the error analysis for the vorticity scheme is carried out first. As a byproduct of these error bounds and the problem decoupling, the convergence rates for the pressure and velocity are readily obtained in the natural norms with constants independent of the viscosity. We also present details about how the analysis of the method is modified for axisymmetric, meridian Brinkman flows; and modify the decoupling strategy to incorporate the case of Dirichlet boundary conditions for the velocity. A set of numerical examples in two and three spatial dimensions illustrate the robustness and accuracy of the finite element method, as well as its competitive computational cost compared to recent fully-mixed and augmented formulations of incompressible flows.
\end{abstract}

Keywords: Brinkman equations; vorticity formulation; decoupling of unknowns; finite elements; axisymmetric domains; error analysis; optimal convergence; generalized Stokes problem.

Mathematical subject classification 2000: . 65N30, 65N12, 76D07, 65N15.

\section{Introduction}

The numerical solution of incompressible flow problems (Stokes, Navier-Stokes, Stokes-Darcy) formulated in terms of the vorticity, velocity, and pressure fields has been carried out using diverse discretization techniques going from spectral to discontinuous Galerkin, fully-mixed, augmented finite elements and axisymmetric formulations (see e.g. Bernardi \& Chorfi, 2007, Cockburn \& Cui, 2012, Amara et al. 2007, Ern et al. 1999 , Amoura et al. 2007, Salaün \& Salmon, 2015, Anaya et al. 2016a, Alvarez et al. 2016 and the references therein).

Of particular interest for us, is the linear Brinkman problem, which stands as a suitable framework for

\footnotetext{
${ }^{\dagger}$ Email: vanaya@ubiobio.cl

$\ddagger$ Corresponding author. Email: dmora@ubiobio.cl

Email: ruizbaier@maths.ox.ac.uk
}

(c) The author 2016. Published by Oxford University Press on behalf of the Institute of Mathematics and its Applications. All rights reserved. 
the study of Stokes and Darcy flows (cf. Guzmán \& Neilan 2012), as well as semi-discretizations of transient Stokes equations. For the velocity-vorticity-pressure formulation of this problem, in Anaya et al. (2015a) the authors proposed augmented mixed formulations based on Raviart-Thomas finite elements for the velocity and piecewise continuous finite elements for the vorticity and pressure, also deriving solvability and error estimates using the Lax-Milgram theory. On the other hand, a dual mixed formulation (in 3D) has been introduced and analyzed in Vassilevski \& Villa (2014), where the well-posedness of the continuous and discrete formulations is established by virtue of the Babuška-Brezzi theory. The Galerkin scheme in this case is based on Nédélec, Raviart-Thomas and piecewise discontinuous finite elements for vorticity, velocity and pressure, respectively. Optimal error estimates and efficient preconditioners were introduced as well. Finally, we mention the stabilized mixed method analyzed for the axisymmetric case in Anaya et al. (2015b), where a priori error estimates are derived uniformly in the viscosity. In contrast with these contributions, in this paper we propose a novel formulation based on two elliptic problems (one for vorticity and the other for pressure) plus a velocity postprocessing. This strategy entails a reduction in computational cost compared with fully-mixed or augmented vorticity-velocity-pressure formulations applied to the same problem. A further feature is that the overall scheme reduces to the solution of two positive definite algebraic systems (see Section 6. A similar decoupling exploiting the momentum equation has been employed for stationary fluid-structure interaction problems (Gatica et al. 2007) and more recently, also in the context of Brinkman flows (Gatica et al. 2014) and the Stokes eigenvalue problem (Meddahi et al. 2015).

Irrespective of the specific form of the flow model, the recovering of accurate flow patterns at an affordable computational burden has proven elusive in the past few decades. Several approaches can be followed, among which one can roughly highlight three main categories (that can be also combined with each other): a) reformulation of the model problem via splitting b) using of high performance computing techniques to achieve satisfactory accuracy for large scale problems, c) introduction of multilevel or multiscale representations of the solutions. Here, we essentially deal with option a), which in principle does not need sophisticated numerical nor refined computational techniques. The idea is related to Uzawa or prediction-correction type methods (Gornak et al. 2013), and is based on decomposing a large saddle point problem into smaller, elliptic ones, but the advantage here is that no splitting error is introduced. Similar strategies (decoupling vorticity from the other fields) also include the immersed boundary method splitting velocity and vorticity proposed in Poncet (2009), and the decoupled vorticity - stream function formulation analyzed in Liu \& E (2001).

Here we will exploit typical regularity assumptions and the boundary conditions to reformulate the coupled problem as two elliptic problems (one for vorticity and the other for pressure) plus a velocity postprocessing. Provided that one does not require to recover velocity patterns directly (common practice in subsurface flow computations, cf. Riaz \& Meiburg. 2003), only the vorticity problem needs to be solved. Alternatively, if only pressure profiles are sought, there is no need to solve the vorticity equations and only a generalized Laplace problem will yield the pressure distribution. The decoupling of unknowns implies in particular that the involved variational formulation can be analyzed using standard tools for elliptic problems, namely the Lax-Milgram Theorem and suitable Céa estimates. We will consider piecewise continuous polynomials of degree $k \geqslant 1$ to approximate vorticity and pressure. Unique solvability of the discrete problems follow by adapting the same tools utilized for the continuous case. Under enough regularity, the finite element scheme converges with optimal rate; such estimates are fully independent of the viscosity (as desired in Brinkmanrelated problems). In addition, we also analyze the continuous and discrete problems written in axisymmetric coordinates. Adequate modifications to the functional framework allow us to consider practically the same arguments as in the Cartesian case. We also investigate the case of Dirichlet boundary conditions for the velocity- a particularly attractive feature, since boundary conditions for the vorticity are no longer required. However, at both continuous and discrete levels, the original system cannot be decoupled into two elliptic problems plus one postprocessing, as before. In this case we write a new formulation as a vorticity-pressure problem, providing the corresponding stability, and error estimates.

The contents of the paper have been structured as follows. Functional spaces and recurrent notation will be collected in the remainder of this Section. The governing equations stated in terms of velocity, vorticity and pressure are presented in Section 2 There, we derive the splitting of the problem into three main parts, and establish their solvability. The approximation via finite elements, the well-posedness of the associated discrete problems, and the corresponding error analysis will be given in Section 3 Remarks on how the analysis is modified in case of axisymmetric formulations are provided in Section 4 Next, the extension to Dirichlet velocities is discussed in Section 5 Several numerical tests illustrating the convergence of the proposed method under different scenarios are reported in Section 6 Finally, we draw some conclusion in Section 7 
Preliminaries

Let $\Omega$ be a bounded domain of $\mathbb{R}^{3}$ with Lipschitz boundary $\Gamma=\partial \Omega$. For any $s \geqslant 0$, the notation $\|\cdot\|_{s, \Omega}$ stands for the norm of the Hilbertian Sobolev spaces $\mathrm{H}^{s}(\Omega)$ or $\mathrm{H}^{s}(\Omega)^{3}$, with the usual convention $\mathrm{H}^{0}(\Omega):=\mathrm{L}^{2}(\Omega)$. For $s \geqslant 0$, we recall the definition of the Hilbert space

$$
\mathrm{H}^{s}(\operatorname{curl} ; \Omega):=\left\{\boldsymbol{\theta} \in \mathrm{H}^{s}(\Omega)^{3}: \operatorname{curl} \boldsymbol{\theta} \in \mathrm{H}^{s}(\Omega)^{3}\right\},
$$

endowed with the norm $\|\boldsymbol{\theta}\|_{\mathrm{H}^{s}(\operatorname{curl} ; \Omega)}^{2}:=\|\boldsymbol{\theta}\|_{s, \Omega}^{2}+\|\operatorname{curl} \boldsymbol{\theta}\|_{s, \Omega}^{2}$, and we will denote $\mathrm{H}(\operatorname{curl} ; \Omega):=\mathrm{H}^{0}(\operatorname{curl} ; \Omega)$.

Moreover, $c$ and $C$, with or without subscripts, tildes, or hats, will represent a generic constant independent of the mesh parameter $h$, assuming different values in different occurrences. In addition, for any vector fields $\boldsymbol{\theta}=\left(\theta_{i}\right)_{i=1,2,3}, \boldsymbol{v}=\left(v_{i}\right)_{i=1,2,3}$ and any scalar field $q$ we recall the notation:

$$
\operatorname{div} \boldsymbol{v}=\partial_{1} v_{1}+\partial_{2} v_{2}+\partial_{3} v_{3}, \boldsymbol{\theta} \times \boldsymbol{v}=\left(\begin{array}{l}
\theta_{2} v_{3}-\theta_{3} v_{2} \\
\theta_{3} v_{1}-\theta_{1} v_{3} \\
\theta_{1} v_{2}-\theta_{2} v_{1}
\end{array}\right), \operatorname{curl} \boldsymbol{v}=\left(\begin{array}{l}
\partial_{2} v_{3}-\partial_{3} v_{2} \\
\partial_{3} v_{1}-\partial_{1} v_{3} \\
\partial_{1} v_{2}-\partial_{2} v_{1}
\end{array}\right), \nabla q=\left(\begin{array}{l}
\partial_{1} q \\
\partial_{2} q \\
\partial_{3} q
\end{array}\right) .
$$

Let us introduce the following functional spaces

$$
\mathbf{Z}:=\{\boldsymbol{\theta} \in \mathrm{H}(\operatorname{curl} ; \Omega): \boldsymbol{\theta} \times \boldsymbol{n}=\mathbf{0} \text { on } \Gamma\} \quad \text { and } \quad \mathrm{Q}:=\mathrm{H}^{1}(\Omega) \cap \mathrm{L}_{0}^{2}(\Omega),
$$

We endow the space $\mathrm{Q}$ with their natural norm:

$$
\|q\|_{\mathrm{Q}}:=\left(\|q\|_{0, \Omega}^{2}+\|\nabla q\|_{0, \Omega}^{2}\right)^{1 / 2} .
$$

However, for the space $\mathbf{Z}$ we will consider the following $\mu$-dependent norm:

$$
\|\boldsymbol{\theta}\|_{\mathbf{z}}:=\left(\|\boldsymbol{\theta}\|_{0, \Omega}^{2}+\mu\|\operatorname{curl} \boldsymbol{\theta}\|_{0, \Omega}^{2}\right)^{1 / 2}
$$

\section{The model problem and well-posedness analysis}

Let us consider the well-known Brinkman problem modelling the steady-state flow of an incompressible viscous fluid within a porous medium. The governing equations can be stated in terms of the velocity $\boldsymbol{u}$, the scaled vorticity $\boldsymbol{\omega}$ and the pressure $p$ of an incompressible viscous fluid (cf. Anaya et al. 2015a. 2016a; Vassilevski $\&$ Villa 2014): Given a sufficiently smooth force density $\boldsymbol{f}$, we seek a triplet $(\boldsymbol{u}, \boldsymbol{\omega}, p)$ such that

$$
\begin{aligned}
\kappa^{-1} \boldsymbol{u}+\sqrt{\mu} \operatorname{curl} \boldsymbol{\omega}+\nabla p & =\boldsymbol{f} & & \text { in } \Omega, \\
\boldsymbol{\omega}-\sqrt{\mu} \operatorname{curl} \boldsymbol{u} & =\mathbf{0} & & \text { in } \Omega, \\
\operatorname{div} \boldsymbol{u} & =0 & & \text { in } \Omega, \\
\boldsymbol{u} \cdot \boldsymbol{n} & =u_{\boldsymbol{n}} & & \text { on } \Gamma, \\
\boldsymbol{\omega} \times \boldsymbol{n} & =\boldsymbol{\omega}_{0} & & \text { on } \Gamma,
\end{aligned}
$$

where $\boldsymbol{u} \cdot \boldsymbol{n}$ stands for the normal component of the velocity, $\mu>0$ is the kinematic viscosity of the fluid, and $\kappa>0$ is the rescaled permeability of the porous skeleton (assumed constant). The boundary conditions considered here are relevant in the context of e.g. geophysical fluids and shallow water models (Karlsen \& Karper 2012)

For the sake of conciseness of the presentation, we will work with homogeneous boundary conditions for the normal velocity and, subsequently, for the vorticity, i.e., $u_{n}=0$, and $\boldsymbol{\omega}_{0}=\mathbf{0}$ on $\Gamma$. Nevertheless, these restrictions do not affect the generality of the forthcoming analysis, which can be further extended to consider other types of boundary conditions, such as e.g. (cf. Vassilevski \& Villa 2014)

$$
\boldsymbol{u} \times \boldsymbol{n}=\boldsymbol{b}, \quad \text { and } p=p_{0} \quad \text { on } \Gamma .
$$

Moreover, if $\Gamma$ admits a disjoint partition $\Gamma=\Gamma_{1} \cup \Gamma_{2}$, the analysis can be also extended to impose for instance 2.4)-(2.5) on $\Gamma_{1}$ and (2.6) on $\Gamma_{2}$. Other alternatives include Groşan et al. (2015), or to consider Robin boundary data as analyzed for velocity-pressure boundary value formulations in Kohr et al. (2014).

The following result is part of Remark 2.5 in Girault \& Raviart (1986). 
LEMMA 2.1 For every $\boldsymbol{v} \in \mathbf{Z}$, it holds that $\operatorname{div}(\operatorname{curl} \boldsymbol{v})=0$ in $\Omega$ and $(\operatorname{curl} \boldsymbol{v}) \cdot \boldsymbol{n}=0$ on $\Gamma$.

We proceed to test 2.2 against a generic $\boldsymbol{\theta} \in \mathbf{Z}$. Then, integrating by parts and using the boundary conditions, we arrive at

$$
\int_{\Omega} \boldsymbol{\omega} \cdot \boldsymbol{\theta}-\sqrt{\mu} \int_{\Omega} \boldsymbol{u} \cdot \operatorname{curl} \boldsymbol{\theta}=0 \quad \forall \boldsymbol{\theta} \in \mathbf{Z}
$$

Next, from 2.1 we readily have

$$
\boldsymbol{\kappa}^{-1} \boldsymbol{u}=\boldsymbol{f}-\sqrt{\mu} \operatorname{curl} \boldsymbol{\omega}-\nabla p \quad \text { in } \quad \Omega,
$$

and after replacing 2.8$)$ in 2.7$)$, we obtain

$$
\kappa^{-1} \int_{\Omega} \boldsymbol{\omega} \cdot \boldsymbol{\theta}+\mu \int_{\Omega} \operatorname{curl} \boldsymbol{\omega} \cdot \operatorname{curl} \boldsymbol{\theta}+\sqrt{\mu} \int_{\Omega} \nabla p \cdot \operatorname{curl} \boldsymbol{\theta}=\sqrt{\mu} \int_{\Omega} \boldsymbol{f} \cdot \operatorname{curl} \boldsymbol{\theta} \quad \forall \boldsymbol{\theta} \in \mathbf{Z} .
$$

Finally, using again an integration by parts, the fact that $\boldsymbol{\theta} \in \mathbf{Z}$ and using Lemma 2.1 we can eliminate the pressure term and rewrite 2.9 ) as a variational formulation of the Brinkman problem stated only in terms of the vorticity: Find $\boldsymbol{\omega} \in \mathbf{Z}$ such that

$$
A(\boldsymbol{\omega}, \boldsymbol{\theta})=F(\boldsymbol{\theta}) \quad \forall \boldsymbol{\theta} \in \mathbf{Z},
$$

where the bilinear form $A: \mathbf{Z} \times \mathbf{Z} \rightarrow \mathbb{R}$ and the linear functional $F: \mathbf{Z} \rightarrow \mathbb{R}$ are defined by

$$
A(\boldsymbol{\omega}, \boldsymbol{\theta}):=\kappa^{-1} \int_{\Omega} \boldsymbol{\omega} \cdot \boldsymbol{\theta}+\mu \int_{\Omega} \operatorname{curl} \boldsymbol{\omega} \cdot \operatorname{curl} \boldsymbol{\theta}, \quad F(\boldsymbol{\theta}):=\sqrt{\mu} \int_{\Omega} \boldsymbol{f} \cdot \operatorname{curl} \boldsymbol{\theta} .
$$

Now we establish the unique solvability of 2.10 .

THEOREM 2.1 (Vorticity solution) Assume that $f \in L^{2}(\Omega)^{3}$. Then, there exists a unique solution $\boldsymbol{\omega} \in \mathbf{Z}$ to 2.10. Moreover, there exists $C>0$ such that

$$
\|\boldsymbol{\omega}\|_{\mathbf{Z}} \leqslant C\|\boldsymbol{f}\|_{0, \Omega}
$$

Proof. First, we observe that the bilinear form $A$, and the linear functional $F$ are bounded with a constant independent of $\mu$. More precisely, there exist $C_{1}, C_{2}>0$ such that

$$
|A(\boldsymbol{\omega}, \boldsymbol{\theta})| \leqslant C_{1}\|\boldsymbol{\omega}\|_{\mathbf{Z}}\|\boldsymbol{\theta}\|_{\mathbf{Z}} \quad \text { and } \quad|F(\boldsymbol{\theta})| \leqslant C_{2}\|\boldsymbol{\theta}\|_{\mathbf{Z}}
$$

In addition, is straightforward to see that $A(\cdot, \cdot)$ is elliptic over the whole space $\mathbf{Z}$. Therefore, the result follows as a direct consequence of the Lax-Milgram Theorem.

REMARK 2.1 (Pressure solve) Let us notice that, as a consequence of the generalized Poincaré inequality and the Lax-Milgram Theorem, the pressure field can be computed as the unique solution of the following problem: Find $p \in \mathbf{Q}$ such that

$$
\int_{\Omega} \nabla p \cdot \nabla q=\int_{\Omega} f \cdot \nabla q \quad \forall q \in \mathrm{Q} .
$$

This variational formulation has been obtained by testing 2.1) with $\nabla q$ for $q \in \mathrm{Q}$ and using integration by parts, combined with 2.3 and the boundary conditions $[2.4,2.5$. Moreover, the following continuous dependence holds: there exists $C>0$ such that

$$
\|p\|_{\mathrm{Q}} \leqslant C\|\boldsymbol{f}\|_{0, \Omega} \text {. }
$$

REMARK 2.2 (Boundary data) As mentioned in the introduction, if instead 2.4 - 2.5) we consider Dirichlet conditions for the velocity, then the problem -written in the specific form $2.1-2.3$ - cannot be completely split into 2.10 and 2.11) using classical tools. Moreover, it is not clear how to analyze that problem without compromising the regularity of solutions and the convergence of the discrete schemes, as discussed in Arnold $e t$ al. (2012) for the approximation of the vector Laplacian, and in Anaya et al. (2016a); Vassilevski \& Villa (2014) for Brinkman equations. However, as we will see in Section 5 Dirichlet velocities can be directly incorporated after reformulating the problem as a coupled vorticity-pressure system.

\section{Finite element approximation}

In this section, we introduce the Galerkin scheme of problem 2.10 and analyze its well-posedness by establishing suitable assumptions on the finite element subspaces involved. Error estimates are also derived. 


\subsection{Formulation and solvability}

Let $\left\{\mathscr{T}_{h}(\Omega)\right\}_{h>0}$ be a shape-regular family of partitions of the polyhedral region $\bar{\Omega}$, by tetrahedrons $T$ of diameter $h_{T}$, with mesh size $h:=\max \left\{h_{T}: T \in \mathscr{T}_{h}(\Omega)\right\}$. In what follows, given an integer $k \geqslant 1$ and a subset $S$ of $\mathbb{R}^{3}, \mathscr{P}_{k}(S)$ denotes the space of polynomial functions defined in $S$ of total degree $\leqslant k$.

Moreover, for any $T \in \mathscr{T}_{h}(\Omega)$, we introduce the following local space (local Nédélec space):

$$
\mathbb{N}_{k}(T):=\mathscr{P}_{k-1}(T) \oplus \mathscr{P}_{k-1}(T) \times \boldsymbol{x}
$$

where $\boldsymbol{x}$ is a generic vector of $\mathbb{R}^{3}$, and let us define the following finite element subspaces:

$$
\begin{array}{ll}
\mathbf{Z}_{h}:=\left\{\boldsymbol{\theta}_{h} \in \mathbf{Z}:\left.\boldsymbol{\theta}_{h}\right|_{T} \in \mathbb{N}_{k}(T)\right. & \left.\forall T \in \mathscr{T}_{h}(\Omega)\right\}, \\
\mathrm{Q}_{h}:=\left\{q_{h} \in \mathrm{Q}:\left.q_{h}\right|_{T} \in \mathscr{P}_{k}(T)\right. & \left.\forall T \in \mathscr{T}_{h}(\Omega)\right\} .
\end{array}
$$

Then, the Galerkin scheme associated with the continuous variational formulation 2.10 reads as follows: Find $\boldsymbol{\omega}_{h} \in \mathbf{Z}_{h}$ such that

$$
\kappa^{-1} \int_{\Omega} \omega_{h} \cdot \theta_{h}+\mu \int_{\Omega} \operatorname{curl} \omega_{h} \cdot \operatorname{curl} \theta_{h}=\sqrt{\mu} \int_{\Omega} f \cdot \operatorname{curl} \theta_{h} \quad \forall \theta_{h} \in \mathbf{Z}_{h}
$$

We are now in a position to state the main result of this section which yields the solvability of the discrete vorticity Brinkman problem 3.3 and the corresponding Céa estimate.

THEOREM 3.1 (Discrete vorticity solvability) Let $k \geqslant 1$ be an integer and let $\mathbf{Z}_{h}$ be given by 3.1. Then, there exists a unique solution $\boldsymbol{\omega}_{h} \in \mathbf{Z}_{h}$ to problem 3.3 and there exists a positive constant $C>0$ such that the following continuous dependence result holds:

$$
\left\|\boldsymbol{\omega}_{h}\right\|_{\mathbf{z}} \leqslant C\|\boldsymbol{f}\|_{0, \Omega} .
$$

Moreover, there exists a constant $\widehat{C}>0$ such that

$$
\left\|\boldsymbol{\omega}-\boldsymbol{\omega}_{h}\right\|_{\mathbf{Z}} \leqslant \widehat{C} \inf _{\boldsymbol{\theta}_{h} \in \mathbf{Z}_{h}}\left\|\boldsymbol{\omega}-\boldsymbol{\theta}_{h}\right\|_{\mathbf{Z}}
$$

where $C$ and $\widehat{C}$ are independent of $\mu$ and $h$, and $\boldsymbol{\omega} \in \mathbf{Z}$ is the unique solution to problem (2.10).

Now, we introduce the finite element discretization of 2.11: Find $p_{h} \in \mathrm{Q}_{h}$ such that

$$
\int_{\Omega} \nabla p_{h} \cdot \nabla q_{h}=\int_{\Omega} f \cdot \nabla q_{h} \quad \forall q_{h} \in \mathrm{Q}_{h} .
$$

The following result establishes the unique solvability of the discrete pressure problem $(3.5)$ and the corresponding Céa estimate.

THEOREM 3.2 (Discrete pressure solvability) Let $k \geqslant 1$ be an integer and let $\mathrm{Q}_{h}$ be given by 3.2. Then, there exists a unique solution $p_{h} \in \mathrm{Q}_{h}$ to problem (3.5) and there exists a positive constant $C>0$ such that the following continuous dependence result holds:

$$
\left\|p_{h}\right\|_{\mathrm{Q}} \leqslant C\|\boldsymbol{f}\|_{0, \Omega} .
$$

Moreover, there exists a constant $\widehat{C}>0$ such that

$$
\left\|p-p_{h}\right\|_{\mathrm{Q}} \leqslant \widehat{C} \inf _{q_{h} \in \mathrm{Q}_{h}}\left\|p-q_{h}\right\|_{\mathrm{Q}}
$$

where $C$ and $\widehat{C}$ are independent of $\mu$ and $h$, and $p \in \mathrm{Q}$ is the unique solution to problem 2.11).

\subsection{Convergence analysis}

According to 3.4 and 3.6 , it only remains to prove that $\boldsymbol{\omega}$ and $p$ can be conveniently approximated by functions in $\mathbf{Z}_{h}$ and $\mathbf{Q}_{h}$, respectively. With this purpose, we introduce for $s>1 / 2$, the Nédeléc global interpolation operator $\mathscr{R}_{h}: \mathrm{H}^{s}($ curl; $\Omega) \cap \mathbf{Z} \rightarrow \mathbf{Z}_{h}$ (see e.g. Alonso \& Valli. 1999). This map satisfies the following property. 
Lemma 3.1 For all $\boldsymbol{\theta} \in \mathrm{H}^{s}(\mathbf{c u r l} ; \Omega), s \in(1 / 2, k]$ there exists $C>0$, independent of $h$, such that

$$
\left\|\boldsymbol{\theta}-\mathscr{R}_{h} \boldsymbol{\theta}\right\|_{\mathbf{Z}} \leqslant C h^{s}\|\boldsymbol{\theta}\|_{\mathrm{H}^{s}(\mathbf{c u r l} ; \Omega)} .
$$

Now, for all $s>1 / 2$, let $\Pi: \mathrm{H}^{1+s}(\Omega) \cap \mathrm{Q} \rightarrow \mathrm{Q}_{h}$ be the usual Lagrange interpolant, for which the following error estimate is available:

LEMmA 3.2 For all $q \in \mathrm{H}^{1+s}(\Omega), s \in(1 / 2, k]$ there exists $C>0$, independent of $h$, such that

$$
\left\|q-\Pi_{h} q\right\|_{\mathrm{Q}} \leqslant C h^{s}\|q\|_{\mathrm{H}^{1+s}(\Omega)} .
$$

We now turn to the statement of convergence properties of the discrete problems 3.3 and 3.5 .

THEOREM 3.3 (Convergence of vorticity and pressure) Let $k \geqslant 1$ and let $\mathbf{Z}_{h}$ and $\mathrm{Q}_{h}$ be given by (3.1) and (3.2), respectively. Let $\boldsymbol{\omega} \in \mathbf{Z}$, and $\boldsymbol{\omega}_{h} \in \mathbf{Z}_{h}$ be the unique solutions to the continuous and discrete problems 2.10) and (3.3), respectively, and let $p \in \mathrm{Q}$, and $p_{h} \in \mathrm{Q}_{h}$ be the unique solutions to the continuous and discrete problems 2.11 and (3.5), respectively. Assume that $\boldsymbol{\omega} \in \mathrm{H}^{s}(\operatorname{curl} ; \Omega)$, and $p \in \mathrm{H}^{1+s}(\Omega)$, for some $s \in(1 / 2, k]$. Then, there exists $C>0$ independent of $h$ and $\mu$ such that

$$
\left\|\boldsymbol{\omega}-\boldsymbol{\omega}_{h}\right\|_{\mathbf{Z}} \leqslant C h^{s}\|\boldsymbol{\omega}\|_{\mathrm{H}^{s}(\mathbf{c u r l} ; \Omega)}, \quad\left\|p-p_{h}\right\|_{\mathrm{Q}} \leqslant C h^{s}\|p\|_{\mathrm{H}^{1+s}(\Omega)} .
$$

Proof. The proof follows from 3.4, 3.6, and error estimates from Lemmas 3.1 and 3.2

\subsection{Recovering the velocity field}

Solution of 2.10) delivers the vorticity field, whereas the pressure can be obtained from 2.11). In addition, it is possible to readily obtain the remaining quantity of interest in 2.1 -2.5 (the velocity $\boldsymbol{u}$ ): If $\boldsymbol{\omega} \in \mathbf{Z}$ and $p \in \mathrm{Q}$ are the unique solutions of 2.10 and 2.11, respectively. Then, according to 2.8 we have that

$$
\boldsymbol{u}=\kappa(\boldsymbol{f}-\sqrt{\mu} \operatorname{curl} \boldsymbol{\omega}-\nabla p)
$$

At the discrete level, this strategy corresponds to computing the velocity as a post-processing of vorticity and pressure: If $\boldsymbol{\omega}_{h} \in \mathbf{Z}_{h}$ and $p_{h} \in \mathrm{Q}_{h}$ are the unique solutions of (3.3) and 3.5, respectively, then the function

$$
\boldsymbol{u}_{h}:=\kappa\left(\mathscr{P}_{h} \boldsymbol{f}-\sqrt{\mu} \operatorname{curl} \boldsymbol{\omega}_{h}-\nabla p_{h}\right)
$$

is an approximation of the velocity, where $\mathscr{P}_{h}: \mathrm{L}^{2}(\Omega)^{3} \rightarrow U_{h}:=\left\{\boldsymbol{v}_{h} \in \mathrm{L}^{2}(\Omega)^{3}:\left.v_{h}\right|_{T} \in \mathscr{P}_{k-1}(T)^{3} \quad \forall T \in\right.$ $\left.\mathscr{T}_{h}(\Omega)\right\}$ is the $\mathrm{L}^{2}$-orthogonal projector. Then, for any $s \in(0, k]$,

$$
\left\|\boldsymbol{v}-\mathscr{P}_{h} \boldsymbol{v}\right\|_{0, \Omega} \leqslant C h^{S}\|\boldsymbol{v}\|_{s, \Omega}
$$

We note $\boldsymbol{u}_{h}$ is element-wise discontinuous. If other properties at the discrete level are sought (such as locally divergence-free), further projection steps are required.

The accuracy of such approximation is established in the following result.

THEOREM 3.4 (Convergence of velocity) Let $\boldsymbol{\omega} \in \mathbf{Z}$ and $p \in \mathrm{Q}$ be the unique solutions of 2.10) and 2.11, respectively, and $\boldsymbol{\omega}_{h} \in \mathbf{Z}_{h}$ and $p_{h} \in \mathrm{Q}_{h}$ be the unique solutions of (3.3) and (3.5), respectively. Assume that $\boldsymbol{\omega} \in \mathrm{H}^{S}(\operatorname{curl} ; \Omega), p \in \mathrm{H}^{1+s}(\Omega)$ and $\boldsymbol{f} \in \mathrm{H}^{S}(\Omega)^{3}$, for some $s \in(1 / 2, k]$. Then, there exists $C>0$ independent of $h$ and $\mu$ such that

$$
\left\|\boldsymbol{u}-\boldsymbol{u}_{h}\right\|_{0, \Omega} \leqslant C h^{s}\left(\|\boldsymbol{f}\|_{\mathrm{H}^{s}(\Omega)}+\|\boldsymbol{\omega}\|_{\mathrm{H}^{s}(\mathbf{c u r l} ; \Omega)}+\|p\|_{\mathrm{H}^{1+s}(\Omega)}\right) .
$$

Proof. From 3.7 and 3.8, and the triangle inequality, it follows that

$$
\left\|\boldsymbol{u}-\boldsymbol{u}_{h}\right\|_{0, \Omega} \leqslant \kappa\left(\left\|\boldsymbol{f}-\mathscr{P}_{h} \boldsymbol{f}\right\|_{0, \Omega}+\left\|\sqrt{\mu} \operatorname{curl}\left(\boldsymbol{\omega}_{h}-\boldsymbol{\omega}\right)\right\|_{0, \Omega}+\left\|\nabla\left(p-p_{h}\right)\right\|_{0, \Omega}\right) .
$$

Then, the result follows from Theorem 3.3 and 3.9 . 

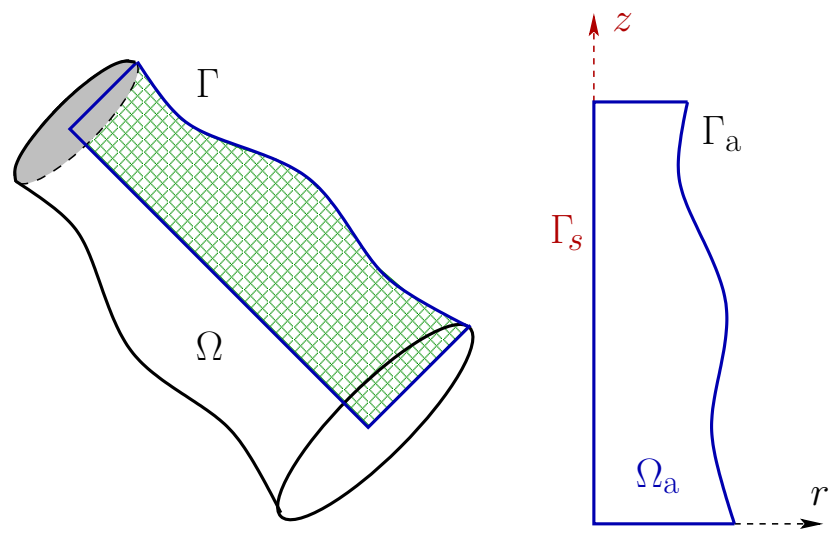

FIG. 1. Sketch of a full three dimensional domain $\Omega$ with boundary $\Gamma$ and the axisymmetric meridional domain $\Omega_{\mathrm{a}}$ with boundary $\Gamma_{\mathrm{a}}$ (left and right, respectively). Here $\Gamma_{s}$ stands for the symmetry axis.

\section{The axisymmetric case}

Let us now assume that the forcing term in 2.1 , the domain $\Omega$, and the non-swirling flow patterns are all symmetric with respect to a given axis $\Gamma_{s}$, lying on $r=0$. Therefore, system 2.1)- 2.5) can be recast as the following axisymmetric problem, with unknowns $u_{r}, u_{z}, \omega$ and $p$ (radial and vertical velocity components, scalar vorticity and pressure), defined in the meridional domain $\Omega_{\mathrm{a}}$ (see Figure 1 ):

$$
\begin{aligned}
& \kappa^{-1} \boldsymbol{u}+\sqrt{\mu} \operatorname{curl}_{\mathbf{a}} \omega+\nabla p=\boldsymbol{f} \text { in } \Omega_{\mathrm{a}}, \\
& \omega-\sqrt{\mu} \operatorname{rot} \boldsymbol{u}=0 \quad \text { in } \Omega_{\mathrm{a}}, \\
& \operatorname{div}_{\mathrm{a}} \boldsymbol{u}=0 \quad \text { in } \Omega_{\mathrm{a}}, \\
& \boldsymbol{u} \cdot \boldsymbol{n}=0 \quad \text { on } \Gamma_{\mathrm{a}}, \\
& \omega=0 \quad \text { on } \Gamma_{\mathrm{a}}
\end{aligned}
$$

where the additional differential operators acting on vectors $\boldsymbol{v}=\left(v_{r}, v_{z}\right)$ and scalars $\varphi$ read

$$
\operatorname{div}_{\mathrm{a}} \boldsymbol{v}:=\partial_{z} v_{z}+r^{-1} \partial_{r}\left(r v_{r}\right), \quad \operatorname{rot} \boldsymbol{v}:=\partial_{r} v_{z}-\partial_{z} v_{r}, \quad \operatorname{curl}_{\mathbf{a}} \varphi:=\left(\partial_{z} \varphi,-r^{-1} \partial_{r}(r \varphi)\right)^{T} .
$$

An augmented mixed finite element scheme for the problem above has been recently analyzed in Anaya $e t$ al. (2015b), where a priori error estimates are derived uniformly in the viscosity (see also a variant applied to the stream-function-vorticity formulation in Anaya et al. 2016b). As we will see, following the decoupling strategy implemented in Sections 2 and 3 will yield a computationally attractive alternative to that formulation. First, we recall some notation and relations of weighted spaces, specialized to our needs.

By $\operatorname{L}_{\alpha}^{p}\left(\Omega_{\mathrm{a}}\right)$ we denote the weighted Lebesgue space of measurable functions $\varphi$ for which

$$
\|\varphi\|_{\mathrm{L}_{\alpha}^{p}\left(\Omega_{\mathrm{a}}\right)}^{p}:=\int_{\Omega_{\mathrm{a}}}|\varphi|^{p} r^{\alpha} \mathrm{d} r \mathrm{~d} z<\infty,
$$

and by $\mathrm{L}_{1,0}^{2}\left(\Omega_{\mathrm{a}}\right)$ we denote the restriction of $\mathrm{L}_{1}^{2}\left(\Omega_{\mathrm{a}}\right)$ to functions with zero weighted integral. Moreover, the space $\mathrm{H}_{r}^{k}\left(\Omega_{\mathrm{a}}\right)$ consists of all functions in $\mathrm{L}_{1}^{2}\left(\Omega_{\mathrm{a}}\right)$ whose derivatives up to order $k$ are also in $\mathrm{L}_{1}^{2}\left(\Omega_{\mathrm{a}}\right)$, and related norms and semi-norms are defined in the standard way. In particular,

$$
|\varphi|_{\mathrm{H}_{1}^{1}\left(\Omega_{\mathrm{a}}\right)}^{2}:=\int_{\Omega_{\mathrm{a}}}\left(\left|\partial_{r} \varphi\right|^{2}+\left|\partial_{z} \varphi\right|^{2}\right) r \mathrm{~d} r \mathrm{~d} z,
$$

and the space $\widetilde{\mathrm{H}}_{1}^{1}\left(\Omega_{\mathrm{a}}\right):=\mathrm{H}_{1}^{1}\left(\Omega_{\mathrm{a}}\right) \cap \mathrm{L}_{-1}^{2}\left(\Omega_{\mathrm{a}}\right)$, endowed with the $\mu$-dependent norm

$$
\|\varphi\|_{\widetilde{\mathrm{H}}_{1}^{1}\left(\Omega_{\mathrm{a}}\right)}:=\left(\|\varphi\|_{\mathrm{L}_{1}^{2}\left(\Omega_{\mathrm{a}}\right)}^{2}+\mu|\varphi|_{\mathrm{H}_{1}^{1}\left(\Omega_{\mathrm{a}}\right)}^{2}+\mu\|\varphi\|_{\mathrm{L}_{-1}^{2}\left(\Omega_{\mathrm{a}}\right)}^{2}\right)^{1 / 2},
$$

is a Hilbert space. We will also require the following weighted scalar and vectorial functional spaces:

$$
\mathbf{Z}^{\mathrm{a}}:=\left\{\varphi \in \widetilde{\mathrm{H}}_{1}^{1}\left(\Omega_{\mathrm{a}}\right) ; \varphi=0 \text { on } \Gamma_{\mathrm{a}}\right\}, \quad \mathrm{Q}^{\mathrm{a}}:=\mathrm{H}_{1}^{1}\left(\Omega_{\mathrm{a}}\right) \cap \mathrm{L}_{1,0}^{2}\left(\Omega_{\mathrm{a}}\right),
$$




$$
\mathbf{H}\left(\operatorname{div}_{\mathrm{a}}, \Omega_{\mathrm{a}}\right):=\left\{\boldsymbol{v} \in \mathrm{L}_{1}^{2}\left(\Omega_{\mathrm{a}}\right)^{2} ; \operatorname{div}_{\mathrm{a}} \boldsymbol{v} \in \mathrm{L}_{1}^{2}\left(\Omega_{\mathrm{a}}\right)\right\}, \quad \mathbf{H}\left(\operatorname{rot}, \Omega_{\mathrm{a}}\right):=\left\{\boldsymbol{v} \in \mathrm{L}_{1}^{2}\left(\Omega_{\mathrm{a}}\right)^{2} ; \operatorname{rot} \boldsymbol{v} \in \mathrm{L}_{1}^{2}\left(\Omega_{\mathrm{a}}\right)\right\} .
$$

The spaces $\mathbf{H}\left(\operatorname{div}_{\mathrm{a}}, \Omega_{\mathrm{a}}\right)$ and $\mathrm{H}\left(\operatorname{curl}_{\mathbf{a}}, \Omega_{\mathrm{a}}\right)$ are endowed respectively with the norms:

$$
\|\boldsymbol{v}\|_{\mathbf{H}\left(\operatorname{div}_{\mathrm{a}}, \Omega_{\mathrm{a}}\right)}^{2}:=\|\boldsymbol{v}\|_{\mathrm{L}_{1}^{2}\left(\Omega_{\mathrm{a}}\right)^{2}}^{2}+\left\|\operatorname{div}_{\mathrm{a}} \boldsymbol{v}\right\|_{\mathrm{L}_{1}^{2}\left(\Omega_{\mathrm{a}}\right)}^{2}, \quad\|\varphi\|_{\mathrm{H}\left(\operatorname{curl}_{\mathrm{a}}, \Omega_{\mathrm{a}}\right)}^{2}:=\|\varphi\|_{\mathrm{L}_{1}^{2}\left(\Omega_{\mathrm{a}}\right)}^{2}+\mu\left\|\operatorname{curl}_{\mathbf{a}} \varphi\right\|_{\mathrm{L}_{1}^{2}\left(\Omega_{\mathrm{a}}\right)^{2}}^{2} .
$$

Furthermore, $\|\cdot\|_{\mathrm{H}\left(\operatorname{curl}_{\mathrm{a}}, \Omega_{\mathrm{a}}\right)}$ and $\|\cdot\|_{\widetilde{\mathrm{H}}_{1}^{1}\left(\Omega_{\mathrm{a}}\right)}$ are equivalent norms, and for any $\varphi \in \widetilde{\mathrm{H}}_{1}^{1}\left(\Omega_{\mathrm{a}}\right)$ they verify:

$$
\sqrt{\mu}\left\|\operatorname{curl}_{\mathbf{a}} \varphi\right\|_{L_{1}^{2}\left(\Omega_{\mathrm{a}}\right)^{2}} \leqslant \sqrt{2}\|\varphi\|_{\widetilde{\mathrm{H}}_{1}^{1}\left(\Omega_{\mathrm{a}}\right)}, \quad\|\varphi\|_{\widetilde{\mathrm{H}}_{1}^{1}\left(\Omega_{\mathrm{a}}\right)} \leqslant\|\varphi\|_{\mathrm{H}\left(\operatorname{curl}_{\mathrm{a}}, \Omega_{\mathrm{a}}\right)} \leqslant \sqrt{2}\|\varphi\|_{\widetilde{\mathrm{H}}_{1}^{1}\left(\Omega_{\mathrm{a}}\right)} .
$$

A variational formulation for system 4.1)- 4.5) is derived as in Section 2 . In particular, we repeat the arguments in 2.7)-2.9) together with Lemmas 1.2 and 1.3 from Anaya et al. (2015b), to obtain the following variational formulation: Find $\omega \in \mathbf{Z}^{\mathrm{a}}$ such that

$$
A^{\mathrm{a}}(\omega, \theta)=F^{\mathrm{a}}(\theta) \quad \forall \theta \in \mathbf{Z}^{\mathrm{a}},
$$

where the bilinear form $A^{\mathrm{a}}: \mathbf{Z}^{\mathrm{a}} \times \mathbf{Z}^{\mathrm{a}} \rightarrow \mathbb{R}$ and the linear functional $F^{\mathrm{a}}: \mathbf{Z}^{\mathrm{a}} \rightarrow \mathbb{R}$ are now defined as

$$
A^{\mathrm{a}}(\omega, \theta):=\kappa^{-1} \int_{\Omega_{\mathrm{a}}} \omega \cdot \theta \mathrm{d} r \mathrm{~d} z+\mu \int_{\Omega_{\mathrm{a}}} \operatorname{curl}_{\mathbf{a}} \omega \cdot \operatorname{curl}_{\mathbf{a}} \theta \mathrm{d} r \mathrm{~d} z, \quad F^{\mathrm{a}}(\theta):=\sqrt{\mu} \int_{\Omega_{\mathrm{a}}} \boldsymbol{f} \cdot \operatorname{curl}_{\mathbf{a}} \theta \mathrm{d} r \mathrm{~d} z .
$$

In a similar manner, the pressure $p \in \mathrm{Q}^{\mathrm{a}}$ can be computed from:

$$
\int_{\Omega_{\mathrm{a}}} \nabla p \cdot \nabla q \mathrm{~d} r \mathrm{~d} z=\int_{\Omega_{\mathrm{a}}} \boldsymbol{f} \cdot \nabla q \mathrm{~d} r \mathrm{~d} z \quad \forall q \in \mathrm{Q}^{\mathrm{a}} .
$$

The well-posedness analysis of (4.7) and 4.8, can be treated in the same way as done in Section 2 as a consequence of 4.6 and the Lax-Milgram Theorem, problem 4.7) admits a unique solution. Moreover, there exists $C>0$ independent of $\mu$ such that

$$
\|\omega\|_{\mathbf{Z}^{\mathrm{a}}} \leqslant C\|\boldsymbol{f}\|_{0, \Omega_{\mathrm{a}}} .
$$

Analogously, the unique solvability of problem 4.8 derives from the generalized Poincaré inequality and the Lax-Milgram Theorem. Also the following continuous dependence holds: there exists $C>0$ independent of $\mu$ such that

$$
\|p\|_{\mathrm{Q}^{\mathrm{a}}} \leqslant C\|\boldsymbol{f}\|_{0, \Omega_{\mathrm{a}}} .
$$

Introducing the finite element subspaces (for any $k \geqslant 1$ )

$$
\begin{aligned}
& \mathbf{Z}_{h}^{\mathrm{a}}:=\left\{\theta_{h} \in \mathbf{Z}^{\mathrm{a}}:\left.\theta_{h}\right|_{T} \in \mathscr{P}_{k}(T) \forall T \in \mathscr{T}_{h}\left(\Omega_{\mathrm{a}}\right)\right\}, \\
& \mathrm{Q}_{h}^{\mathrm{a}}:=\left\{q_{h} \in \mathrm{Q}^{\mathrm{a}}:\left.q_{h}\right|_{T} \in \mathscr{P}_{k}(T) \forall T \in \mathscr{T}_{h}\left(\Omega_{\mathrm{a}}\right)\right\},
\end{aligned}
$$

we can write a Galerkin scheme associated to 4.7 : Find $\omega \in \mathbf{Z}_{h}^{\mathrm{a}}$ such that

$$
A^{\mathrm{a}}\left(\omega_{h}, \theta_{h}\right)=F^{\mathrm{a}}\left(\theta_{h}\right) \quad \forall \theta_{h} \in \mathbf{Z}_{h}^{\mathrm{a}},
$$

whereas the discrete counterpart of 4.8 reads: Find $p_{h} \in \mathrm{Q}_{h}^{\mathrm{a}}$ such that

$$
\int_{\Omega_{\mathrm{a}}} \nabla p_{h} \cdot \nabla q_{h} \mathrm{~d} r \mathrm{~d} z=\int_{\Omega_{\mathrm{a}}} f \cdot \nabla q_{h} \mathrm{~d} r \mathrm{~d} z \quad \forall q_{h} \in \mathrm{Q}_{h}^{\mathrm{a}} .
$$

Additionally, as in Section 3.3 we can compute continuous and discrete velocities using

$$
\begin{aligned}
\boldsymbol{u} & =\kappa\left(\boldsymbol{f}-\sqrt{\mu} \operatorname{curl}_{\mathbf{a}} \omega-\nabla p\right), \\
\boldsymbol{u}_{h} & :=\kappa\left(\mathscr{P}_{h} \boldsymbol{f}-\sqrt{\mu} \operatorname{curl}_{\mathbf{a}} \omega_{h}-\nabla p_{h}\right) .
\end{aligned}
$$

Well-posedness and error estimates for 4.11) and 4.12) can be established following the lines of Section 3 Moreover, Mercier \& Raugel 1982. Lemma 6.3 implies that the following estimate holds (see also Belhachmi et al. 2006).

LEMmA 4.1 There exists $C>0$, independent of $h$ and $\mu$, such that for all $\theta \in \mathrm{H}_{1}^{k+1}\left(\Omega_{\mathrm{a}}\right)$ :

$$
\left\|\theta-\Pi_{h} \theta\right\|_{\widetilde{\mathrm{H}}_{1}^{1}\left(\Omega_{\mathrm{a}}\right)} \leqslant C h^{k}\|\theta\|_{\mathrm{H}_{1}^{k+1}\left(\Omega_{\mathrm{a}}\right)},
$$

where $\Pi_{h}: \widetilde{\mathrm{H}}_{1}^{1}\left(\Omega_{\mathrm{a}}\right) \cap \mathrm{H}_{1}^{2}\left(\Omega_{\mathrm{a}}\right) \rightarrow \mathbf{Z}_{h}^{\mathrm{a}}$ is the Lagrange interpolator of a sufficiently smooth $\theta$. 
THEOREM 4.1 (Convergence of vorticity, pressure and velocity) Let $k \geqslant 1$ and let $\mathbf{Z}_{h}^{\mathrm{a}}$ and $\mathrm{Q}_{h}^{\mathrm{a}}$ be given by (4.9) and 4.10), respectively. Let $\omega \in \mathbf{Z}^{\mathrm{a}}$, and $\omega_{h} \in \mathbf{Z}_{h}^{\mathrm{a}}$ be the unique solutions to the continuous and discrete problems 4.7) and (4.11, respectively, and let $p \in \mathrm{Q}^{\mathrm{a}}$, and $p_{h} \in \mathrm{Q}_{h}^{\mathrm{a}}$ be the unique solutions to the continuous and discrete problems 4.8 and 4.12 , respectively. Assume that $\omega \in \mathrm{H}_{1}^{k+1}\left(\Omega_{\mathrm{a}}\right)$, and $p \in \mathrm{H}_{1}^{k+1}\left(\Omega_{\mathrm{a}}\right)$. Then, there exists $C>0$ independent of $h$ and $\mu$ such that

$$
\left\|\omega-\omega_{h}\right\|_{\mathbf{Z}^{\mathrm{a}}}+\left\|p-p_{h}\right\|_{\mathrm{Q}^{\mathrm{a}}}+\left\|\boldsymbol{u}-\boldsymbol{u}_{h}\right\|_{0, \Omega_{\mathrm{a}}} \leqslant C h^{k}\left(\|\omega\|_{\mathrm{H}_{1}^{k+1}\left(\Omega_{\mathrm{a}}\right)}+\|p\|_{\mathrm{H}_{1}^{k+1}\left(\Omega_{\mathrm{a}}\right)}\right) .
$$

Proof. The proof follows from a Céa estimate, Lemma 4.1. and 4.13-4.14.

\section{The case of no-slip velocity boundary conditions}

As mentioned in Remark 2.2 the case of Dirichlet data for the velocity cannot be readily analyzed under the same framework of Sections 2 and 3 Here we rewrite the Brinkman equations as a vorticity-pressure system, followed by the postprocess (3.7), 3.8) to recover the velocity.

\subsection{A new vorticity-pressure formulation}

Let us then consider 2.1-2.3 equipped with

$$
\boldsymbol{u}=\mathbf{0} \quad \text { on } \Gamma,
$$

as the only boundary condition. An advantage of fixing also the tangent component of the velocity on the boundary (giving therefore no-slip velocities) is that no boundary conditions need to be imposed on the vorticity.

Testing 2.2 against $\boldsymbol{\theta} \in \widehat{\mathbf{Z}}:=\mathrm{H}(\mathbf{c u r l} ; \Omega)$, integrating by parts, using [5.1, and combining the result with 2.1, gives

$$
\kappa^{-1} \int_{\Omega} \boldsymbol{\omega} \cdot \boldsymbol{\theta}+\mu \int_{\Omega} \operatorname{curl} \boldsymbol{\omega} \cdot \operatorname{curl} \boldsymbol{\theta}+\sqrt{\mu} \int_{\Omega} \nabla p \cdot \operatorname{curl} \boldsymbol{\theta}=\sqrt{\mu} \int_{\Omega} \boldsymbol{f} \cdot \operatorname{curl} \boldsymbol{\theta} \quad \forall \boldsymbol{\theta} \in \widehat{\mathbf{Z}} .
$$

Note that 5.2] almost coincides with 2.9], except for the test space, which was $\mathbf{Z}=\{\boldsymbol{\theta} \in \mathrm{H}(\mathbf{c u r l} ; \Omega): \boldsymbol{\theta} \times \boldsymbol{n}=$ $\mathbf{0}$ on $\Gamma$ \}.

We next proceed to test 2.1) against $\nabla q \in L^{2}(\Omega)^{3}$, we integrate by parts, and apply (2.3) and 5.1) to end up with

$$
\sqrt{\mu} \int_{\Omega} \operatorname{curl} \omega \cdot \nabla q+\int_{\Omega} \nabla p \cdot \nabla q=\int_{\Omega} f \cdot \nabla q \quad \forall q \in \mathrm{Q} .
$$

In this way, problem 2.1 -2.3, , 5.1) is equivalent to the following variational formulation: Find $(\boldsymbol{\omega}, p) \in \widehat{\mathbf{Z}} \times \mathrm{Q}$ such that

$$
\mathscr{A}((\boldsymbol{\omega}, p),(\boldsymbol{\theta}, q))=F(\boldsymbol{\theta}, q) \quad \forall(\boldsymbol{\theta}, q) \in \widehat{\mathbf{Z}} \times \mathbf{Q},
$$

where the bilinear form $\mathscr{A}:(\widehat{\mathbf{Z}} \times \mathrm{Q}) \times(\widehat{\mathbf{Z}} \times \mathrm{Q}) \rightarrow \mathbb{R}$ and linear functional $\mathscr{F}: \widehat{\mathbf{Z}} \times \mathrm{Q} \rightarrow \mathbb{R}$ are now specified as

$$
\begin{aligned}
\mathscr{A}((\boldsymbol{\omega}, p),(\boldsymbol{\theta}, q)) & :=\kappa^{-1} \int_{\Omega} \boldsymbol{\omega} \cdot \boldsymbol{\theta}+\int_{\Omega}(\sqrt{\mu} \operatorname{curl} \boldsymbol{\omega}+\nabla p) \cdot(\sqrt{\mu} \operatorname{curl} \boldsymbol{\theta}+\nabla q), \\
\mathscr{F}(\boldsymbol{\theta}, q) & :=\int_{\Omega} \boldsymbol{f} \cdot(\sqrt{\mu} \operatorname{curl} \boldsymbol{\theta}+\nabla q) .
\end{aligned}
$$

\subsection{The Galerkin method}

Let us now introduce a conforming finite element subspace for vorticity

$$
\widehat{\mathbf{Z}}_{h}:=\left\{\boldsymbol{\theta}_{h} \in \widehat{\mathbf{Z}}:\left.\boldsymbol{\theta}_{h}\right|_{T} \in \mathbb{N}_{k}(T) \quad \forall T \in \mathscr{T}_{h}(\Omega)\right\},
$$

while $\mathrm{Q}_{h}$, as defined in 3.2, will be used for the pressure approximation. The finite element counterpart of 5.3) then reads: Find $\left(\boldsymbol{\omega}_{h}, p_{h}\right) \in \widehat{\mathbf{Z}}_{h} \times \mathrm{Q}_{h}$ such that

$$
\mathscr{A}\left(\left(\boldsymbol{\omega}_{h}, p_{h}\right),\left(\boldsymbol{\theta}_{h}, q_{h}\right)\right)=\mathscr{F}\left(\boldsymbol{\theta}_{h}, q_{h}\right) \quad \forall\left(\boldsymbol{\theta}_{h}, q_{h}\right) \in \widehat{\mathbf{Z}}_{h} \times \mathrm{Q}_{h} .
$$

We readily have that this problem is well-posed. 
THEOREM 5.1 The method (5.4) is well defined.

Proof. Since (5.4) is a square linear system, it is enough to prove uniqueness. To this end, we assume that $\boldsymbol{f}=\mathbf{0}$ and we test the problem with $\left(\boldsymbol{\theta}_{h}, q_{h}\right)=\left(\boldsymbol{\omega}_{h}, p_{h}\right)$ to obtain

$$
\kappa^{-1}\left\|\boldsymbol{\omega}_{h}\right\|_{0, \Omega}^{2}+\left\|\sqrt{\mu} \operatorname{curl} \boldsymbol{\omega}_{h}+\nabla p_{h}\right\|_{0, \Omega}^{2}=0,
$$

showing that $\boldsymbol{\omega}_{h}=\mathbf{0}$ and $p_{h}=0$.

\subsection{Error analysis}

In this section we prove error estimates for vorticity and pressure. We start by writing the following error equation:

$$
\mathscr{A}\left(\left(\boldsymbol{\omega}-\boldsymbol{\omega}_{h}, p-p_{h}\right),\left(\boldsymbol{\theta}_{h}, q_{h}\right)\right)=0 \quad \forall\left(\boldsymbol{\theta}_{h}, q_{h}\right) \in \widehat{\mathbf{Z}}_{h} \times \mathrm{Q}_{h} .
$$

Moreover, for all $(\boldsymbol{\theta}, q) \in \widehat{\mathbf{Z}} \times \mathrm{Q}$, we have that there exist $C_{1}, C_{2}>0$ such that

$$
C_{1}\left(\|\boldsymbol{\theta}\|_{0, \Omega}^{2}+\|\sqrt{\mu} \operatorname{curl} \boldsymbol{\omega}+\nabla q\|_{0, \Omega}^{2}+\|q\|_{0, \Omega}^{2}\right) \leqslant \mathscr{A}((\boldsymbol{\theta}, q),(\boldsymbol{\theta}, q)) \leqslant C_{2}\left(\|\boldsymbol{\theta}\|_{0, \Omega}^{2}+\|\sqrt{\mu} \operatorname{curl} \boldsymbol{\omega}+\nabla q\|_{0, \Omega}^{2}+\|q\|_{0, \Omega}^{2}\right) .
$$

We have the following theorem.

THEOREM 5.2 Assume that $\boldsymbol{\omega} \in \mathrm{H}^{s}(\operatorname{curl} ; \Omega)$, and $p \in \mathrm{H}^{1+s}(\Omega)$, for some $s \in(1 / 2, k]$. Then, there exists $C>0$, independent of $h$ and $\mu$, such that

$$
\left\|\boldsymbol{\omega}-\boldsymbol{\omega}_{h}\right\|_{0, \Omega}+\left\|\sqrt{\mu} \operatorname{curl}\left(\boldsymbol{\omega}-\boldsymbol{\omega}_{h}\right)+\nabla\left(p-p_{h}\right)\right\|_{0, \Omega}+\left\|p-p_{h}\right\|_{0, \Omega} \leqslant C h^{s}\left(\|\boldsymbol{\omega}\|_{\mathrm{H}^{s}(\mathbf{c u r l} ; \Omega)}+\|p\|_{\mathrm{H}^{1+s}(\Omega)}\right) .
$$

Proof. The result is a consequence of [5.5, 5.6, together with Lemmas 3.1 and 3.2

Finally, if we define again $\boldsymbol{u}$ and $\boldsymbol{u}_{h}$ as in (3.7) and (3.8), respectively; then Theorem 5.2 provides an identical result as the one given in Theorem 3.4 regarding the accuracy of the discrete velocity.

REMARK 5.1 Note that the new method (5.4) (solving the Brinkman problem under Dirichlet boundary conditions for velocity) is designed for the $3 \mathrm{D}$ case, but its modification to the $2 \mathrm{D}$ case follows straightforwardly. It suffices to notice that the vorticity is now a scalar function, whose natural approximation space corresponds to the conforming space

$$
\widehat{\mathbf{Z}}_{h}:=\left\{\boldsymbol{\theta}_{h} \in \mathrm{H}^{1}(\Omega):\left.\boldsymbol{\theta}_{h}\right|_{T} \in \mathscr{P}_{k}(T) \quad \forall T \in \mathscr{T}_{h}(\Omega)\right\} .
$$

In this case, the computational cost of (5.4) (in its lowest order configuration) is almost $2 N_{v}$, where $N_{v}$ denotes the number of nodes in the triangulation, thus providing a very competitive alternative in comparison to other classical techniques based on velocity-pressure formulations (see Mardal et al. 2002).

\section{Numerical results}

In what follows, we present a set of numerical examples illustrating our findings from the performance of the FE method described in Sections 35 These tests confirm the theoretical error bounds (here obtained for FE families with $k=1$ and $k=2$ ).

\subsection{Example 1: convergence history in $2 D$}

The first test consists of approximating a manufactured solution on a two-dimensional domain. We consider $\Omega=(-1,1)^{2}, \kappa^{-1}=50, \mu=0.001$, and construct the forcing term $f$ so that the exact solution to 2.1)- 2.3 is given by the following smooth functions

$$
\left.\boldsymbol{u}=\left(\begin{array}{c}
\sin (\pi x) \cos (\pi y) \\
-\cos (\pi x) \sin (\pi y)
\end{array}\right) \text { (satisfying } 2.4\right) \text { ), } \omega=2 \sqrt{\mu} \pi \sin (\pi x) \sin (\pi y) \text { (satisfying } 2.5 \text { ), } p=x^{4}-y^{4} \in \mathrm{Q} \text {. }
$$

The overall algorithm employed in all tests of this section consists in first solving (3.3), next (3.5), and finally applying the postprocess 3.8. In the Darcy limit $(\mu \rightarrow 0)$ one realizes that the specific problem definition implies that only the last two steps of the algorithm are needed. For $k=1$ the velocity is projected on piecewise constant functions, whereas for $k=2$ we utilize discontinuous, piecewise linear elements. We proceed 

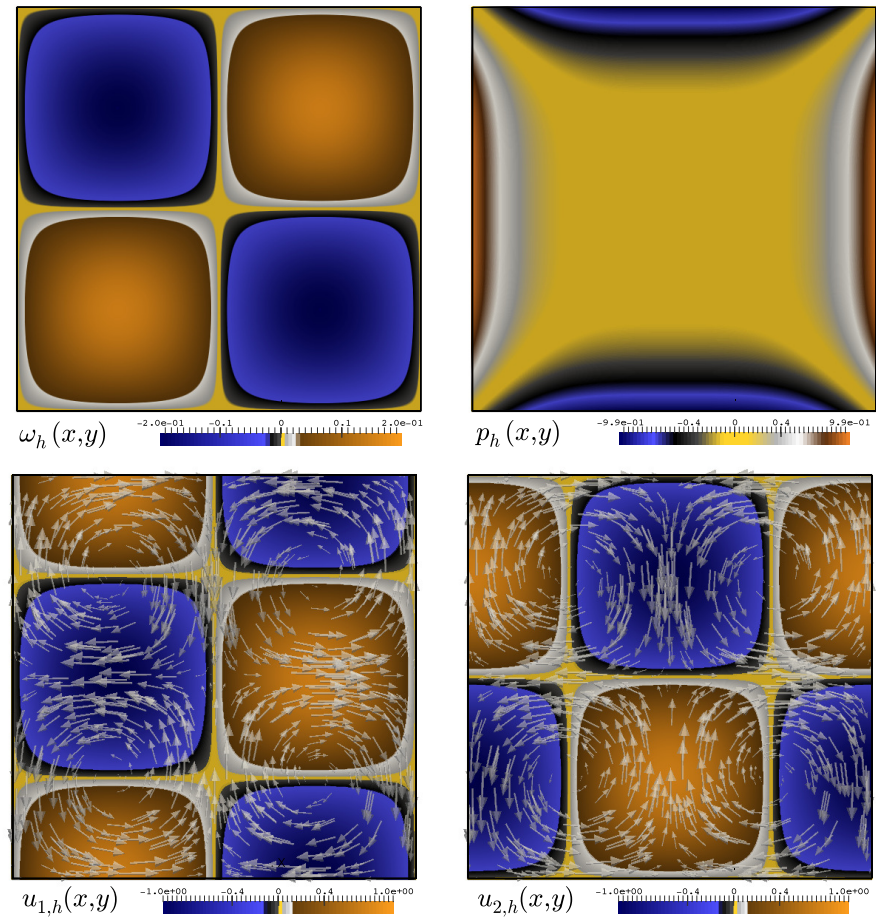

FIG. 2. Example 1: approximated vorticity, pressure distribution, and postprocessed velocity components.

TABLE 1. Example 1: convergence tests against analytical solutions on a sequence of uniformly refined triangulations of the domain $\Omega=(-1,1)^{2}$.

\begin{tabular}{ccccccr}
\hline$h$ & $\mathrm{e}(\omega)$ & \multicolumn{1}{c}{$r(\omega)$} & $\mathrm{e}(p)$ & $r(p)$ & $\mathrm{e}(\boldsymbol{u})$ & $r(\boldsymbol{u})$ \\
\hline \multicolumn{7}{c}{ Approximation with $k=1$} \\
7.4142 & $8.08 \mathrm{e}-1$ & - & 3.2341 & - & 1.3693 & - \\
$3.54 \mathrm{e}-1$ & $7.41 \mathrm{e}-1$ & 0.1252 & 2.0411 & 0.6654 & 1.0403 & 0.3832 \\
$1.77 \mathrm{e}-1$ & $3.69 \mathrm{e}-1$ & 1.0053 & 1.0805 & 0.9205 & $5.47 \mathrm{e}-1$ & 0.9298 \\
$8.84 \mathrm{e}-2$ & $1.77 \mathrm{e}-1$ & 1.0604 & $5.45 \mathrm{e}-1$ & 0.9801 & $2.77 \mathrm{e}-1$ & 0.9822 \\
$4.42 \mathrm{e}-2$ & $8.72 \mathrm{e}-2$ & 1.0199 & $2.74 \mathrm{e}-1$ & 0.9950 & $1.39 \mathrm{e}-1$ & 0.9956 \\
$2.21 \mathrm{e}-2$ & $4.34 \mathrm{e}-2$ & 1.0063 & $1.37 \mathrm{e}-1$ & 0.9987 & $6.95 \mathrm{e}-2$ & 0.9989 \\
$1.10 \mathrm{e}-2$ & $2.17 \mathrm{e}-2$ & 1.0022 & $6.85 \mathrm{e}-2$ & 0.9996 & $3.47 \mathrm{e}-2$ & 0.9997 \\
$5.52 \mathrm{e}-3$ & $1.08 \mathrm{e}-2$ & 1.0007 & $3.42 \mathrm{e}-2$ & 0.9999 & $1.74 \mathrm{e}-2$ & 0.9999 \\
\hline & $5.42 \mathrm{e}-2$ & 1.0002 & $1.71 \mathrm{e}-2$ & 0.9999 & $8.69 \mathrm{e}-3$ & 0.9999 \\
\hline 1.4142 & $6.30 \mathrm{e}-2$ & \multicolumn{7}{c}{ Approximation with $k=2$} & & \\
$7.07 \mathrm{e}-1$ & $1.93 \mathrm{e}-2$ & 1.7053 & 1.5366 & - & - \\
$3.54 \mathrm{e}-1$ & $5.31 \mathrm{e}-3$ & 1.8630 & $8.91 \mathrm{e}-1$ & 2.1479 & $3.13 \mathrm{e}-1$ & 1.4819 \\
$1.77 \mathrm{e}-1$ & $1.35 \mathrm{e}-3$ & 1.9779 & $2.26 \mathrm{e}-2$ & 1.9558 & $8.40 \mathrm{e}-2$ & 1.8983 \\
$8.84 \mathrm{e}-2$ & $3.35 \mathrm{e}-4$ & 2.0052 & $5.68 \mathrm{e}-3$ & 1.9810 & $2.14 \mathrm{e}-2$ & 1.9744 \\
$4.42 \mathrm{e}-2$ & $8.30 \mathrm{e}-5$ & 2.0013 & $1.42 \mathrm{e}-3$ & 1.9962 & $5.37 \mathrm{e}-3$ & 1.9935 \\
$2.21 \mathrm{e}-2$ & $2.11 \mathrm{e}-5$ & 1.9996 & $3.56 \mathrm{e}-4$ & 1.9981 & $3.36 \mathrm{e}-3$ & 1.9983 \\
$1.10 \mathrm{e}-2$ & $6.01 \mathrm{e}-6$ & 1.9998 & $8.92 \mathrm{e}-5$ & 1.9987 & $8.42 \mathrm{e}-5$ & 1.9995 \\
$5.52 \mathrm{e}-3$ & $2.13 \mathrm{e}-6$ & 1.9907 & $2.30 \mathrm{e}-5$ & 1.9986 & $2.14 \mathrm{e}-5$ & 1.9998 \\
\hline
\end{tabular}

to construct a series of uniformly successively refined triangular meshes for $\Omega$ and compute grid-dependent experimental errors and convergence rates defined by

$$
e(\boldsymbol{\omega})=\left\|\boldsymbol{\omega}-\boldsymbol{\omega}_{h}\right\|_{\mathbf{Z}}, \quad e(p)=\left\|p-p_{h}\right\|_{\mathrm{Q}}, \quad e(\boldsymbol{u})=\left\|\boldsymbol{u}-\boldsymbol{u}_{h}\right\|_{0, \Omega}, \quad r(\cdot)=\log (e(\cdot) / \hat{e}(\cdot))[\log (h / \hat{h})]^{-1},
$$



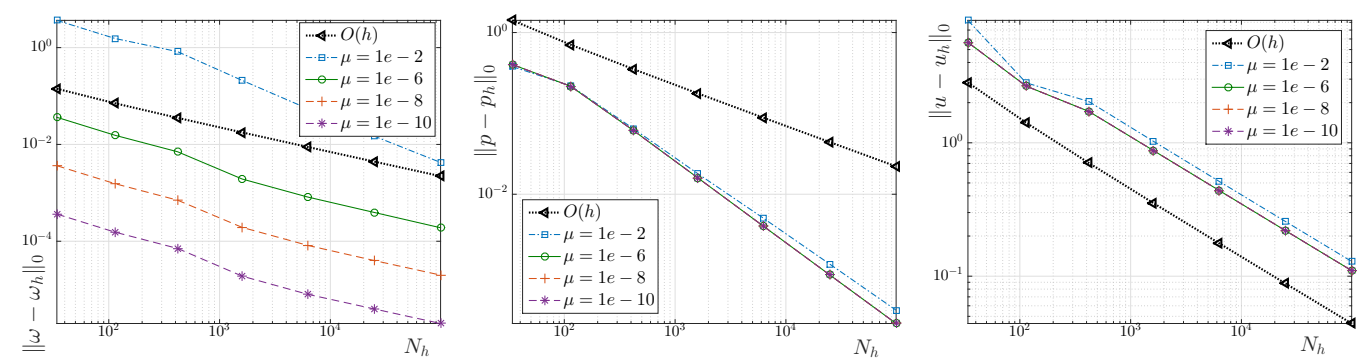

FIG. 3. Example 2: accuracy test in case of Dirichlet boundary conditions for the velocity. Error histories for vorticity (left), pressure (middle), and velocity (right) measured in the $L^{2}-$ norm.

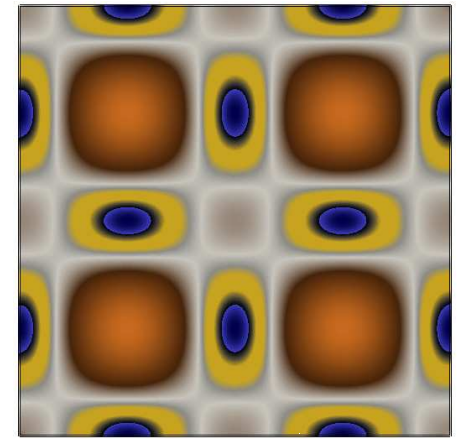

$\omega_{h}(x, y)$

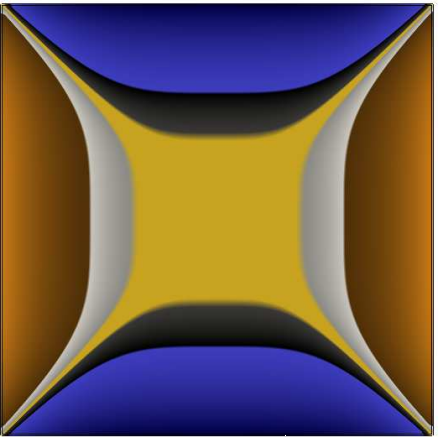

$p_{h}(x, y)$

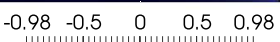

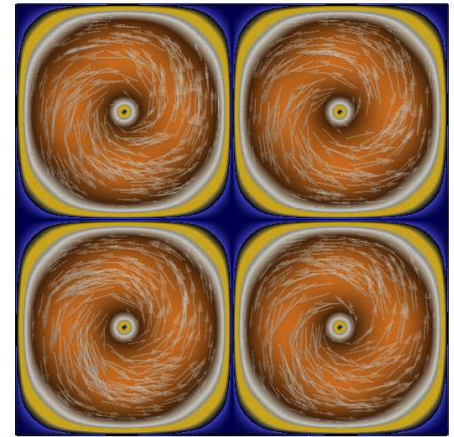

$\left|u_{h}(x, y)\right|$

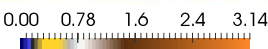

FIG. 4. Example 2: accuracy test in case of Dirichlet boundary conditions for the velocity. Computed vorticity (left), pressure (middle), and velocity (right).

where $e, \hat{e}$ denote errors computed on two consecutive meshes of sizes $h, \hat{h}$, respectively. The error history and approximate solutions computed at the finest mesh are collected in Table 1 and Figure 2 These indicate optimal accuracy for $k=1$ and $k=2$, according to Theorems 3.3 3.4

\subsection{Example 2: Dirichlet velocity conditions}

Next we investigate the accuracy of formulation (5.4), valid for no-slip velocities on $\Gamma$. The domain is again the square $\Omega=(-1,1)^{2}$ and we fix the permeability $\kappa=1$ with the aim of also testing the robustness of the error estimates with respect to viscosity. A manufactured solution satisfying 5.1 is given by

$$
\boldsymbol{u}=\left(\begin{array}{c}
2 \pi \sin ^{2}(\pi x) \sin (\pi y) \cos (\pi y) \\
-2 \pi \sin (\pi x) \cos (\pi x) \sin ^{2}(\pi y)
\end{array}\right), \omega=2 \sqrt{\mu} \pi^{2}\left(\sin ^{2}(\pi x)\left[4 \sin ^{2}(\pi y)-1\right]-\sin ^{2}(\pi y)\right), p=x^{4}-y^{4}
$$

We perform a series of convergence histories (focusing on the lowest order method, $k=1$ ), on a sequence of nested structured meshes and using $\mu \in\{1 e-2,1 e-4,1 e-6,1 e-8,1 e-10\}$. The results are collected in Figure 3 , where the plot on the right panel indicates that for smaller viscosities, the curves practically coincide. On the other hand, independently of the viscosity, we observe that for finer meshes both vorticity and velocity attain the expected $O(h)$ convergence rate predicted by Theorem 5.2 It is also noticed that the pressure error decays much faster, suggesting that the result in Section 5 can be improved. This phenomenon deserves further investigation, but we stress that the overall rate of convergence remains the one given by the theorem. Examples of computed solutions using $\mu=1 e-4$ are depicted in Figure 4 
TABLE 2. Example 3: convergence tests against analytical solutions on a sequence of uniformly refined tetrahedrizations of the domain $\Omega=(0,1) \times(0,1) \times(-1,1)$.

\begin{tabular}{ccccccc}
\hline$h$ & $\mathrm{e}(\boldsymbol{\omega})$ & $r(\boldsymbol{\omega})$ & $\mathrm{e}(p)$ & $r(p)$ & $\mathrm{e}(\boldsymbol{u})$ & $r(\boldsymbol{u})$ \\
\hline $7.07 \mathrm{e}-1$ & 1.0785 & - & 1.5434 & - & 1.0763 & - \\
$3.54 \mathrm{e}-1$ & $5.61 \mathrm{e}-1$ & 0.8872 & $8.32 \mathrm{e}-1$ & 0.8901 & $6.06 \mathrm{e}-1$ & 0.8151 \\
$1.77 \mathrm{e}-1$ & $3.71 \mathrm{e}-1$ & 0.9136 & $4.27 \mathrm{e}-1$ & 0.9613 & $3.16 \mathrm{e}-1$ & 0.9388 \\
$1.01 \mathrm{e}-1$ & $1.91 \mathrm{e}-1$ & 0.9693 & $2.46 \mathrm{e}-1$ & 0.9845 & $1.82 \mathrm{e}-1$ & 0.9825 \\
$6.43 \mathrm{e}-2$ & $1.01 \mathrm{e}-1$ & 0.9396 & $1.57 \mathrm{e}-1$ & 0.9907 & $1.16 \mathrm{e}-1$ & 0.9937 \\
$4.42 \mathrm{e}-2$ & $5.57 \mathrm{e}-2$ & 0.9673 & $1.09 \mathrm{e}-1$ & 0.9902 & $8.02 \mathrm{e}-2$ & 0.9972 \\
$3.21 \mathrm{e}-2$ & $2.74 \mathrm{e}-2$ & 0.9703 & $7.61 \mathrm{e}-2$ & 0.9910 & $5.48 \mathrm{e}-2$ & 0.9976 \\
$1.95 \mathrm{e}-2$ & $1.31 \mathrm{e}-2$ & 0.9741 & $5.32 \mathrm{e}-2$ & 0.9928 & $4.05 \mathrm{e}-2$ & 0.9980 \\
\hline
\end{tabular}

\subsection{Example 3: convergence history in 3D}

The convergence of the method is also tested in a three-dimensional setting. We consider the same values for $\kappa$ and $\mu$ as in the previous test and employ as computational domain the parallelepiped $\Omega=(0,1) \times(0,1) \times$ $(-1,1)$. The following exact solutions are constructed

$$
\boldsymbol{u}=\left(\begin{array}{c}
\sin (\pi x) \cos (\pi y) \cos (\pi z) \\
-2 \cos (\pi x) \sin (\pi y) \cos (\pi z) \\
\cos (\pi x) \cos (\pi y) \sin (\pi z)
\end{array}\right), \boldsymbol{\omega}=\sqrt{\mu}\left(\begin{array}{c}
3 \pi \cos (\pi x) \sin (\pi y) \sin (\pi z) \\
0 \\
-3 \pi \sin (\pi x) \sin (\pi y) \cos (\pi z)
\end{array}\right), p=x^{3}-y^{3}-z^{3},
$$

which also fulfill the boundary data 2.4 - 2.5 and the regularity requirements. The external force $f$ is computed using these functions and 2.1). The same decoupling algorithm is used to generate the error history displayed in Table 2 which puts into evidence optimal convergence rates for all fields. Here we employed the lowest order FE family $k=1$. Iso-surfaces representing the approximate solutions are portrayed in Figure 5

\subsection{Example 4: simulation of axisymmetric flows}

We also carry out a simulation of viscous flow in porous media using an axisymmetric formulation and the method outlined in Section 4 The meridional domain has four sides defined by the symmetry axis (left wall $r=0)$, bottom and top lids $(z=0$ and $z=2$, respectively), and the right side is defined by the parametrization $s \in[0,2], r=2-s / 4+\gamma \cos (\pi s) \sin (\pi s)$, and $z=s-\gamma \cos (\pi s) \sin (\pi s)$, with $\gamma=0.1$ (as sketched in Figure 1). The forcing term and model parameters are

$$
f=\left(-r \sin (\pi r) \cos (\pi z)+4 r^{3}, \pi^{-1} \sin (\pi r) \sin (\pi z)+r \cos (\pi r) \sin (\pi z)-4 z^{3}\right)^{T}, \kappa^{-1}=200, \mu=0.1,
$$

and the boundary data are taken as in 4.4 - 4.5 . A sequence of nine successively refined unstructured triangulations of $\Omega_{\mathrm{a}}$ is generated and, in absence of a known analytical solution, errors are computed with respect to a reference solution $\left(\omega_{\text {ref }}, p_{\text {ref }}, \boldsymbol{u}_{\text {ref }}\right)$ (obtained by solving the decoupled formulation on a highly fine mesh and with $k=2$ ). Experimental errors and convergence rates are shown in Table 3 and indicate optimal accuracy according to Theorem 4.1 Approximate solutions (including rotational extrusion of the axisymmetric velocity and pressure) are illustrated in Figure 6

\subsection{Example 5: viscous flow within a porous tilted cylinder}

We end with the simulation of incompressible flow in a tilted cylindrical column composed of a porous material of low-concentration particles. Even if the non-inclined column has an axisymmetric geometry, the forcing term is more important in the gravity direction and once inclined (forming an angle of $\pi / 4$ between the $z$ and $y$ axes), the geometry loses symmetry with respect to the $z$ axis. Therefore the expected patterns will not be axisymmetric and the Brinkman problem needs to be solved using the full three-dimensional formulation (2.1)-2.5. We construct an unstructured tetrahedral mesh of 71742 vertices and 401115 elements and consider $\kappa=1, \mu=0.01, f=\kappa\left(\exp (-y z)+x \exp \left(-x^{2}\right), \cos (\pi x) \cos (\pi z)-y \exp \left(-y^{2}\right),-10 x y-10 z \exp \left(-z^{2}\right)\right)^{T}$. The numerical solution obtained with a $k=1$ family is presented in Figure 7 

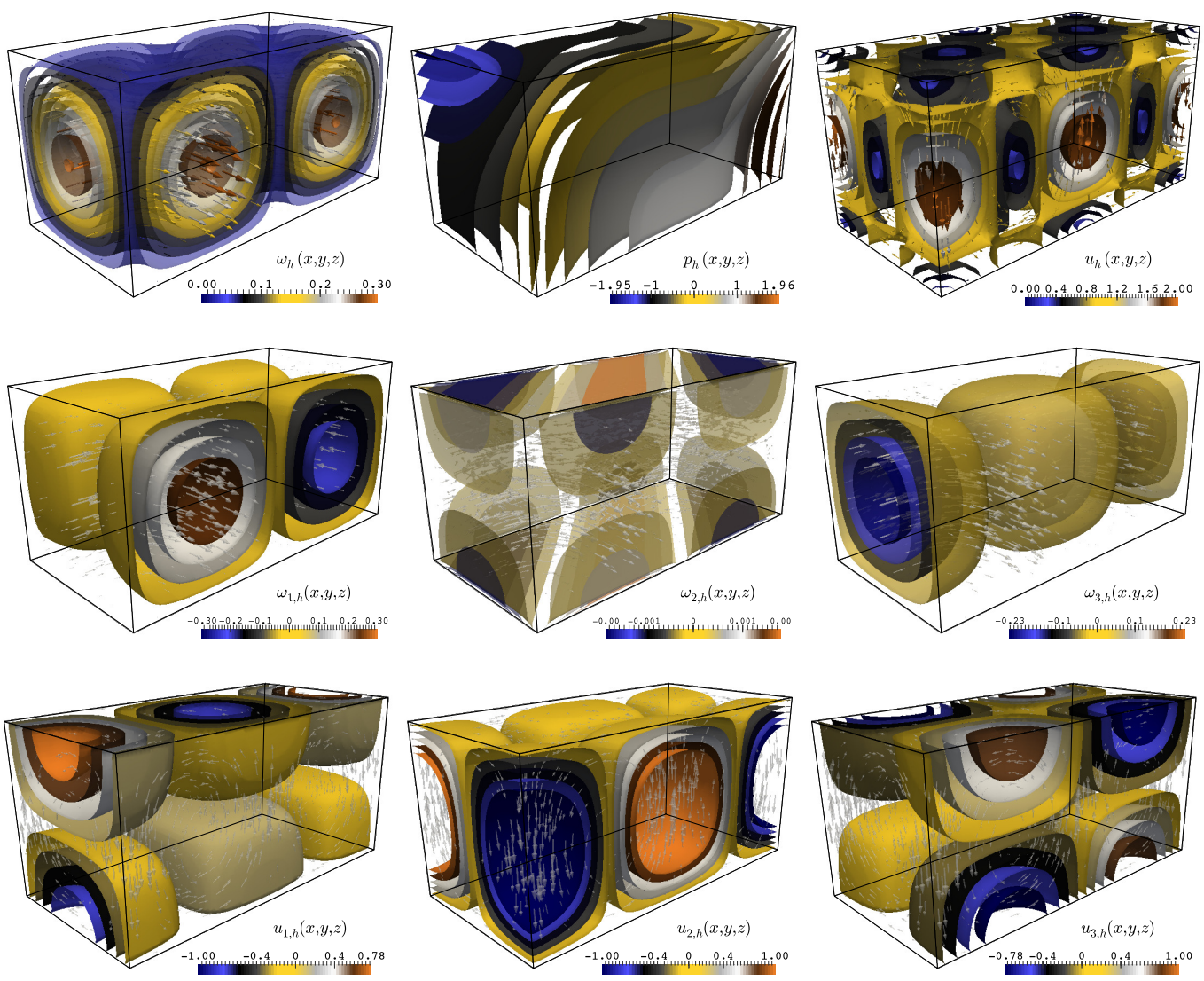

FIG. 5. Example 3: approximated solutions for the accuracy test in $\Omega=(0,1) \times(0,1) \times(-1,1)$. Vorticity magnitude, pressure distribution, velocity magnitude (top row), vorticity components (middle row), and postprocessed velocity components (bottom row).

As mentioned in Section 1, one of the most appealing features of the present method is the low computational cost compared to similarly accurate schemes such as the mixed methods suggested in Alvarez et al. (2016); Vassilevski \& Villa (2014) and the augmented formulation from Anaya et al. (2015a) (see also Section 5 in Alvarez et al. 2016). Without counting the cost of matrix assembly, the most expensive part of the algorithm is the solution of the vorticity problem, which represents only a fraction (roughly a 32\% in 3D, and $20 \%$ in $2 \mathrm{D}$ for $k=1$; and $28 \%$ in 3D, and $16 \%$ in 2D for $k=2$ ) of the matrix size associated to an augmented $\boldsymbol{u}-\boldsymbol{\omega}-p$ formulation exhibiting the same convergence behavior.

\section{Concluding remarks}

In this work, we have presented a new finite element method for the discretization of the vorticity-velocitypressure formulation of the Brinkman equations. The key features of the proposed method are the direct and accurate access to vorticity without invoking postprocessing and its competitive computational cost compared to recent fully-mixed and augmented formulations of incompressible viscous flows. We derived optimal convergence rates (and robust with respect to viscosity) in the natural norms. Some numerical tests have been presented to confirm the theoretically results established, and to illustrate the robustness and efficiency of the proposed method. Possible extensions of this work include the study of vorticity-based formulations of threedimensional Navier-Stokes equations, transient Stokes equations, axisymmetric time-dependent Navier-Stokes equations, and the coupling with Darcy flow and with transport phenomena. 
TABLE 3. Example 4: errors and convergence rates computed on a sequence of unstructured meshes of the axisymmetric domain $\Omega_{\mathrm{a}}$, against a fine reference solution. Errors and convergence rates are re-defined using the axisymmetric spaces.

\begin{tabular}{ccccccr}
\hline$h$ & $\mathrm{e}\left(\boldsymbol{\omega}_{\text {ref }}\right)$ & $r\left(\boldsymbol{\omega}_{\text {ref }}\right)$ & $\mathrm{e}\left(p_{\text {ref }}\right)$ & $r\left(p_{\text {ref }}\right)$ & $\mathrm{e}\left(\boldsymbol{u}_{\text {ref }}\right)$ & $r\left(\boldsymbol{u}_{\text {ref }}\right)$ \\
\hline $5.42 \mathrm{e}-1$ & $1.72 \mathrm{e}-2$ & - & 1.8621 & - & 1.0422 & - \\
$4.25 \mathrm{e}-1$ & $1.41 \mathrm{e}-2$ & 0.8221 & 1.1130 & 1.1169 & $6.19 \mathrm{e}-3$ & 1.1372 \\
$2.48 \mathrm{e}-1$ & $1.17 \mathrm{e}-2$ & 0.3367 & $5.61 \mathrm{e}-1$ & 1.0695 & $3.44 \mathrm{e}-3$ & 1.0918 \\
$1.23 \mathrm{e}-1$ & $8.70 \mathrm{e}-3$ & 0.5231 & $3.08 \mathrm{e}-1$ & 0.9536 & $1.87 \mathrm{e}-3$ & 0.9637 \\
$8.32 \mathrm{e}-2$ & $6.35 \mathrm{e}-3$ & 0.8118 & $1.90 \mathrm{e}-1$ & 1.0418 & $1.19 \mathrm{e}-3$ & 1.0678 \\
$5.47 \mathrm{e}-2$ & $4.37 \mathrm{e}-3$ & 0.8891 & $1.31 \mathrm{e}-1$ & 0.9931 & $8.19 \mathrm{e}-4$ & 0.9845 \\
$3.99 \mathrm{e}-2$ & $3.22 \mathrm{e}-3$ & 0.9706 & $9.03 \mathrm{e}-2$ & 1.0766 & $5.67 \mathrm{e}-4$ & 1.0755 \\
$2.95 \mathrm{e}-2$ & $2.43 \mathrm{e}-3$ & 0.9825 & $6.80 \mathrm{e}-2$ & 0.9929 & $4.26 \mathrm{e}-4$ & 0.9989 \\
$2.42 \mathrm{e}-2$ & $1.90 \mathrm{e}-3$ & 1.0706 & $5.19 \mathrm{e}-2$ & 1.0840 & $3.25 \mathrm{e}-4$ & 1.0844 \\
\hline & \multicolumn{7}{c}{ Approximation with $k=1$} \\
\hline $5.42 \mathrm{e}-1$ & $1.17 \mathrm{e}-2$ & - & $3.64 \mathrm{e}-1$ & - & $3.05 \mathrm{e}-3$ & - \\
$4.25 \mathrm{e}-1$ & $7.78 \mathrm{e}-3$ & 1.7002 & $1.57 \mathrm{e}-1$ & 2.4774 & $1.41 \mathrm{e}-3$ & 2.1625 \\
$2.48 \mathrm{e}-1$ & $4.89 \mathrm{e}-3$ & 0.9627 & $3.78 \mathrm{e}-2$ & 2.3685 & $3.92 \mathrm{e}-4$ & 2.0808 \\
$1.23 \mathrm{e}-1$ & $2.56 \mathrm{e}-3$ & 1.5197 & $1.68 \mathrm{e}-2$ & 2.1512 & $1.20 \mathrm{e}-4$ & 1.9816 \\
$8.32 \mathrm{e}-2$ & $1.28 \mathrm{e}-3$ & 1.7796 & $5.64 \mathrm{e}-3$ & 2.1100 & $4.60 \mathrm{e}-5$ & 2.0921 \\
$5.47 \mathrm{e}-2$ & $6.11 \mathrm{e}-4$ & 1.8622 & $2.65 \mathrm{e}-3$ & 1.8935 & $2.13 \mathrm{e}-5$ & 1.9468 \\
$3.99 \mathrm{e}-2$ & $3.29 \mathrm{e}-4$ & 1.9768 & $1.36 \mathrm{e}-3$ & 2.1204 & $1.09 \mathrm{e}-5$ & 2.0331 \\
$2.95 \mathrm{e}-2$ & $1.87 \mathrm{e}-4$ & 1.9527 & $7.73 \mathrm{e}-4$ & 1.9609 & $6.12 \mathrm{e}-6$ & 1.9649 \\
$2.42 \mathrm{e}-2$ & $1.15 \mathrm{e}-4$ & 2.0559 & $4.87 \mathrm{e}-4$ & 2.0711 & $3.07 \mathrm{e}-6$ & 2.0994 \\
\hline
\end{tabular}
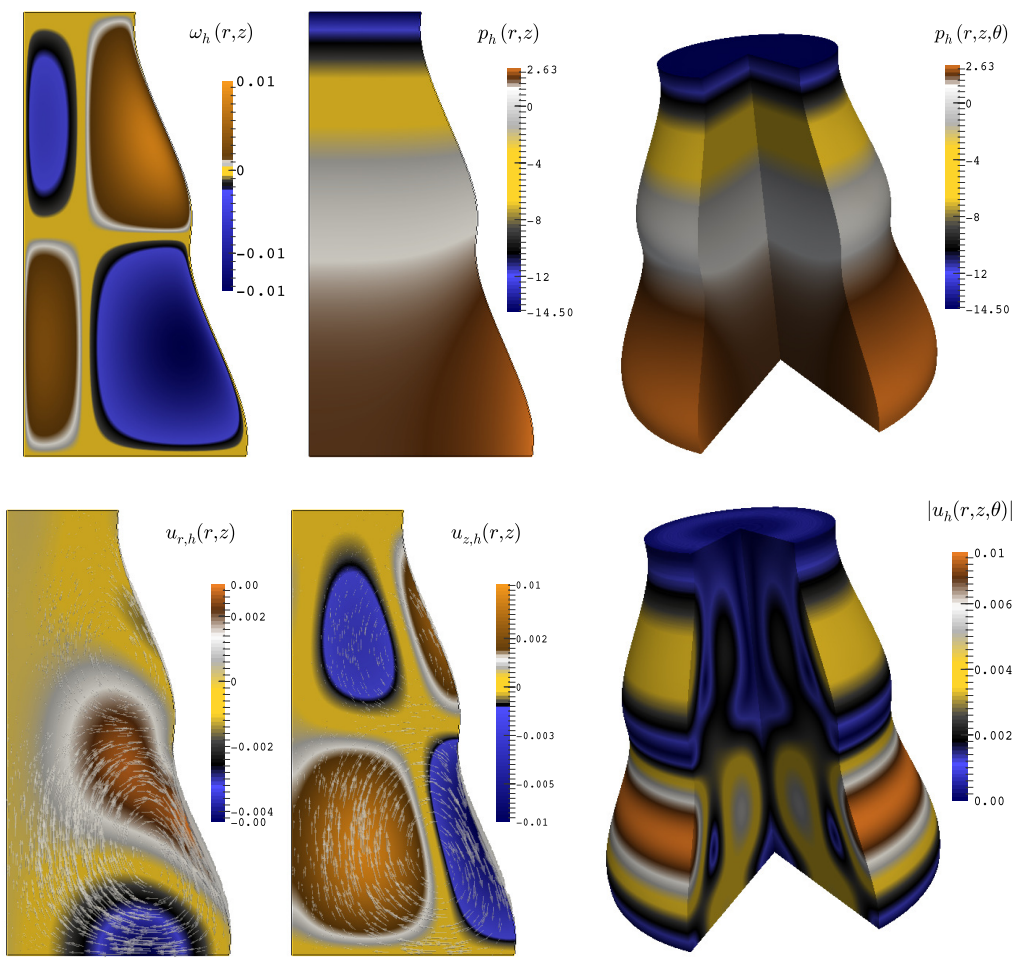

FIG. 6. Example 4: approximated vorticity, pressure distribution, and postprocessed velocity components on the axisymmetric domain $\Omega_{\mathrm{a}}$. 

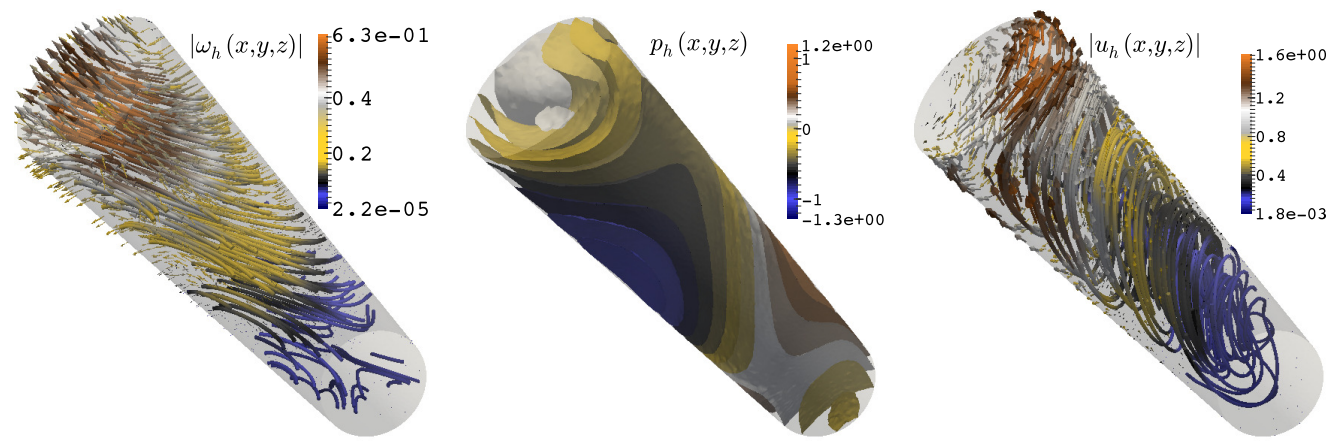

FIG. 7. Example 5: approximated vorticity streamlines and vectors, pressure distribution iso-surfaces, and postprocessed velocity vectors and streamlines.

\section{Acknowledgments}

V. Anaya was partially supported by CONICYT-Chile through project Inserción de Capital Humano Avanzado en la Academia No. 79112012, and DIUBB through project 151408 GI/VC; D. Mora was partially supported by CONICYT-Chile through FONDECYT project 1140791, by Anillo ANANUM, ACT1118, CONICYT (Chile) and by DIUBB through project 151408 GI/VC; and R. Ruiz-Baier acknowledges support by the Elsevier Mathematical Sciences Sponsorship Fund MSSF-201602. This work was advanced during a visit of V. Anaya and D. Mora to the Mathematical Institute of Oxford University.

\section{References}

Alonso, A. \& Valli, A. (1999) An optimal domain decomposition preconditioner for low-frequency time harmonic Maxwell equations. Math. Comp., 68, 607-631.

Alvarez, M., Gatica, G. N. \& Ruiz-BAier, R. (2016) Analysis of a vorticity-based fully-mixed formulation for the 3D Brinkman-Darcy problem. Comput. Methods Appl. Mech. Engrg., 307, 68-95.

Amara, M., Capatina-Papaghiuc, D. \& Trujillo, D. (2007) Stabilized finite element method for Navier-Stokes equations with physical boundary conditions. Math. Comp., 76(259), 1195-1217.

Amoura, K., Azaïez, M., Bernardi, C., Chorfi, N. \& SaAdi, S. (2007) Spectral element discretization of the vorticity, velocity and pressure formulation of the Navier-Stokes problem. Calcolo, 44(3), 165-188.

Anaya, V., Gatica, G. N., Mora, D. \& Ruiz-Baier, R. (2015) An augmented velocity-vorticity-pressure formulation for the Brinkman equations. Int. J. Numer. Meth. Fluids, 79(3), 109-137.

Anaya, V., Mora, D., Oyarzúa, R. \& Ruiz-Baier, R. (2016) A priori and a posteriori error analysis for a mixed scheme for the Brinkman problem. Numer. Math., in press. DOI: 10.1007/s00211-015-0758-X.

Anaya, V., Mora, D., Reales, C. \& Ruiz-Baier, R. (2016) Mixed methods for a stream-functionvorticity formulation of the axisymmetric Brinkman equations. $\mathrm{CI}^{2} \mathrm{MA}$ Pre-print 2016-08, available from http://www.ci2ma.udec.cl.

Anaya, V., Mora, D., Reales, C. \& Ruiz-Baier, R. (2015) Stabilized mixed approximation of axisymmetric Brinkman flows. ESAIM: Math. Model. Numer. Anal., 49(3), 855-874.

Arnold, D. N., Falk, R. S. \& Gopalakrishnan, J. (2012) Mixed finite element approximation of the vector Laplacian with Dirichlet boundary conditions. Math. Models Methods Appl. Sci., 22(9), 1-26.

Belhachmi, Z., Bernardi, C. \& Deparis, S. (2006) Weighted Clément operator and application to the finite element discretization of the axisymmetric Stokes problem. Numer. Math., 105(2), 217-247.

BERNARDI, C. \& CHORFI, N. (2007) Spectral discretization of the vorticity, velocity, and pressure formulation of the Stokes problem. SIAM J. Numer. Anal., 44(2), 826-850. 
CockBURN, B. \& CUI, J. (2012) An analysis of HDG methods for the vorticity-velocity-pressure formulation of the Stokes problem in three dimensions. Math. Comp., 81(279), 1355-1368.

Ern, A., Guermond, J. L. \& QuARTAPelle, L. (1999) Vorticity-velocity formulations of the Stokes problem in 3D. Math. Methods Appl. Sci., 22(6), 531-546.

Gatica, G. N., GAtiCA, L. F. \& MÁrqueZ, A. (2014) Analysis of a pseudostress-based mixed finite element method for the Brinkman model of porous media flow. Numer. Math., 126(4), 635-677.

Gatica, G. N., Márquez, A. \& Meddahi, S. (2007) Analysis of the coupling of primal and dual-mixed finite element methods for a two-dimensional fluid-solid interaction problem. SIAM J. Numer. Anal., 45(5), 2072-2097.

Girault, V. \& Raviart, P. A. (1986) Finite element methods for Navier-Stokes equations. Theory and algorithms. Berlin: Springer-Verlag.

Gornak, T., Guermond, J. L., Iliev, O. \& Minev, P. D. (2013) A direction splitting approach for incompressible Brinkman flow. Int. J. Numer. Anal. Model. B, 4(1), 1-13.

Groşan, T., Kohr, M. \& Wendland, W. L. (2015) Dirichlet problem for a nonlinear generalized DarcyForchheimer-Brinkman system in Lipschitz domains. Math. Methods Appl. Sci., 38(17), 3615-3628.

GuzMán, J. \& NeIlan, M. (2012) A family of nonconforming elements for the Brinkman problem. IMA J. Numer. Anal., 32(4), 1484-1508.

KARlsen, K. H. \& KARPer, T. K. (2012) A convergent mixed method for the Stokes approximation of viscous compressible flow. IMA J. Numer. Anal., 32(3), 725-764.

Kohr, M., LAnZa DE Cristoforis, M. \& Wendland, W. L. (2014) Boundary value problems of Robin type for the Brinkman and Darcy-Forchheimer-Brinkman systems in Lipschitz domains. J. Math. Fluid Mech., 16, 595-630.

LiU, J. G. \& E, W. (2001) Simple finite element method in vorticity formulation for incompressible flows. Math. Comp., 70(234), 579-593.

Meddahi, S., Mora, D. \& Rodríguez, R. (2015) A finite element analysis of a pseudostress formulation for the Stokes eigenvalue problem. IMA J. Numer. Anal., 35(2), 749-766.

MERCIER, B. \& RAUGel, G. (1982) Resolution d'un problème aux limites dans un ouvert axisymétrique par éléments finis en $r$, $z$ et séries de Fourier en $\theta$. RAIRO Anal. Numér., 16, 405-461.

Mardal, K.A., TAI, X.C. \& Winther, R. (2002) A robust finite element method for Darcy-Stokes flow. SIAM J. Numer. Anal., 40(5), 1605-1631.

PoncET, P. (2009) Analysis of an immersed boundary method for three-dimensional flow in vorticity formulation. J. Comput. Phys., 228, 7268-7288.

Riaz, A. \& Meiburg, E. (2003) Three-dimensional miscible displacement simulations in homogeneous porous media with gravity override. J. Fluid Mech., 494, 95-117.

SALAÜN, M. \& SALMON, S. (2015) Low-order finite element method for the well-posed bidimensional Stokes problem. IMA J. Numer. Anal., 35, 427-453.

VAssilevski, P. S. \& Villa, U. (2014) A mixed formulation for the Brinkman problem. SIAM J. Numer. Anal., 52(1), 258-281. 